\title{
The long-wave instability of short-crested waves, via embedding in the oblique two-wave interaction
}

\author{
By THOMAS J. BRIDGES AND FIONA E. LAINE-PEARSON \\ Department of Mathematics and Statistics, University of Surrey, Guildford, Surrey, GU2 7XH, UK
}

(Received 10 October 2003 and in revised form 7 January 2005)

The motivation for this work is the stability problem for short-crested Stokes waves. A new point of view is proposed, based on the observation that an understanding of the linear stability of short-crested waves (SCWs) is closely associated with an understanding of the stability of the oblique non-resonant interaction between two waves. The proposed approach is to embed the SCWs in a six-parameter family of oblique non-resonant interactions. A variational framework is developed for the existence and stability of this general two-wave interaction. It is argued that the resonant SCW limit makes sense a posteriori, and leads to a new stability theory for both weakly nonlinear and finite-amplitude SCWs. Even in the weakly nonlinear case the results are new: transverse weakly nonlinear long-wave instability is independent of the nonlinear frequency correction for SCWs whereas longitudinal instability is influenced by the SCW frequency correction, and, in parameter regions of physical interest there may be more than one unstable mode. With explicit results, a critique of existing results in the literature can be given, and several errors and misconceptions in previous work are pointed out. The theory is developed in some generality for Hamiltonian PDEs. Water waves and a nonlinear wave equation in two space dimensions are used for illustration of the theory.

\section{Introduction}

The aim of this paper is to develop a theory for the long-wave instability of shortcrested Stokes waves (SCWs). These waves are one of the simplest classes of doubly periodic three-dimensional water waves and are therefore of fundamental interest, and they are of practical importance since they appear in models for coastal sand transport, reflection off vertical seawalls, and wave propagation along channels.

Historically, the problem of stability of SCWs has been approached directly. Roskes (1976b) proposed the use of coupled nonlinear Stokes (NLS) equations to model SCW instability, Mollo-Christensen (1981) proposed the use of Whitham modulation theory, Ioualalen \& Kharif (1994) computed eigenvalues of the exact linear stability problem numerically, and Badulin et al. (1995) presented a qualitative analysis based on the Zakharov (1968) Hamiltonian formulation for water waves. As far as we are aware these are the only papers in the literature on a theoretical approach to the linear stability of SCWs.

However, Roberts (1983) points out that SCWs are a special case of two-phase wavetrains, and he proposes that the theory of Ablowitz \& Benney (1970), where modulation equations for multi-phase wavetrains are derived, be used for the stability 


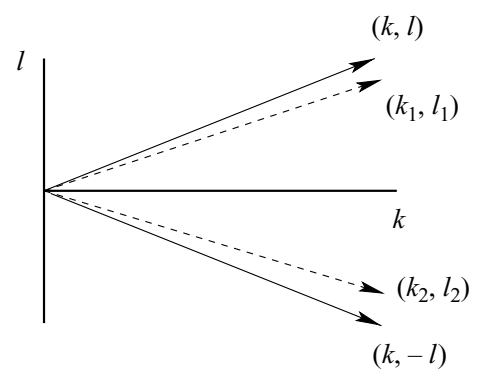

FIGURE 1. Schematic of the deformation of an SCW into a two-wave interaction.

analysis. It is possible that this approach would work, but the Ablowitz-Benney equation is non-local and not easy to work with. However, embedding the SCWs in a two-phase wavetrain turns out to be the correct approach.

The basic observation is that an understanding of the linear stability of SCWs - even weakly nonlinear SCWs - is closely associated with understanding the stability of the oblique non-resonant interaction between two waves. One way to see the connection between SCWs and two-wave interaction is to note that when an SCW becomes unstable to long-wave perturbations, the resonant SCW plus the perturbation is a wave with a wavenumber vector which is no longer resonant.

This observation is a generalization to two space dimensions of the geometry of sideband instability. A weakly nonlinear Stokes wave is stable to perturbations of the same wavelength. However, if a perturbation of a slightly longer wavelength is added, it is unstable. This instability is determined by the susceptibility of the Stokes wave to waves with slightly longer wavelength. In the case of SCWs there are two sidebands: sidebands in wavenumber space associated with both the $x$ and $y$ directions. Therefore the perturbed wavenumber vector is perturbed in both length and direction.

In the theory proposed here, the SCW is first embedded in a six-parameter family of multi-phase wavetrains, as shown schematically in figure 1 , and then a posteriori the limit to resonant SCWs is taken, accumulating along the way enough information to predict all the long-wave instabilities of SCWs. Effectively, the embedding provides information about the susceptibility of the SCW to distortion in wavenumber space by the perturbation.

A by-product of this analysis is a stability theory for the general two-phase wavetrain, which may be of independent interest. For example Onorato et al. (2003) show that the instability of the non-resonant two-wave interaction may explain the doublepeaked power spectrum of waves in shallow water observed by Smith \& Vincent (1992).

The existence of the SCWs is assumed throughout, and attention is restricted to gravity waves on a fluid of infinite depth, although the implications for other classes of waves will be apparent. There is now a range of analytical and numerical existence results in the literature that we can appeal to (e.g. Hsu, Silvester \& Tsuchiya 1980; Roberts 1983; Roberts \& Peregrine 1983; Ioualalen 1993; Kimmoun, Branger \& Kharif 1999; Craig \& Nicholls 2000, 2002 and references therein). A rigorous theory for the existence of gravity SCWs has been elusive and Craig \& Nicholls (2002) point out that there are technical problems associated with small divisors. However, in the case of capillary-gravity SCWs there is a well-developed existence theory (Craig \& Nicholls 2000). 
In addition to the existence of SCWs, the theory also uses an embedding of SCWs in a general non-resonant two-phase wavetrain. Historically, the analyses of twowave and $n$-wave interactions have been considered independently of SCWs. The only results in the literature for the general two-wave interaction are for the weakly nonlinear case (e.g. Longuet-Higgins 1962; Willebrand 1975; Weber \& Barrick 1977; Pierson 1993; Elfouhaily et al. 2000 and references therein). It is easy to show that applying the SCW limit to the (weakly nonlinear) two-wave interaction results in the usual (weakly nonlinear) SCW solutions. However, a key new feature of the theory here is that information is extracted from the embedding, before the limit to SCWs is taken.

In this paper, a theory for the stability of weakly nonlinear SCWs and finiteamplitude SCWs is developed. The only restriction is on the perturbation wavenumbers, which are restricted to long-wave perturbations. These perturbations are generalizations of the Benjamin-Feir instability of plane travelling waves.

A Stokes-type expansion for SCWs is singular in the long-crested limit (see Roberts \& Peregrine 1983, where an alternative theory is proposed which avoids the singularity). Since this paper is primarily concerned with periodic SCWs, it is assumed throughout that the parameter values for the SCWs are chosen away from the long-crested limit. Since the waves under consideration in this paper are in infinite depth, mean flow will be ignored. It is important to note that neglect of mean flow is an assumption. It is certainly true for weakly nonlinear SCWs in infinite depth, but it is an open question whether wave-generated mean flow can occur for finite-amplitude SCWs. The formulation presented here is amenable to including mean flow effects (see discussion in $\S 10$ ). However, it is assumed in this paper that the SCWs are not accompanied by wave-generated mean flow.

The water-wave problem is Hamiltonian, and it will be advantageous to recognize this in the development of the theory. The Hamiltonian approach was first applied by Badulin et al. (1995) to the analysis of SCWs and it was shown to have advantages. This idea is taken a step further here with the use of the multi-symplectic formulation of Hamiltonian PDEs. Since water waves and other Hamiltonian PDEs for SCWs can be reformulated as multi-symplectic systems, a general formulation of multisymplectic Hamiltonian PDEs can be taken as the starting point

$$
\mathbf{M} Z_{t}+\mathbf{K} Z_{x}+\mathbf{L} Z_{y}=\nabla S(Z), \quad Z \in \mathbb{H}
$$

where $\mathbf{M}, \mathbf{K}$ and $\mathbf{L}$ are constant skew-symmetric operators, $\mathbb{H}$ is a linear space (either $\mathbb{R}^{n}$ for the nonlinear wave equation or an inner-product space of functions on the cross-section for water waves), and the gradient of $S$ is with respect to the inner product on $\mathbb{H}$. Details of this formulation for water waves and other Hamiltonian PDEs can be found in Bridges (1996, 1997a,b) and the details needed here are recorded in $\S 2$, including a new multi-symplectic formulation of the nonlinear wave equation in two space dimensions. This nonlinear wave equation provides a simple model problem for SCWs and an example where the long-wave transverse instability of SCWs can disappear at low amplitude.

The advantage of the multi-symplectic framework is five-fold: $(a)$ it is clear and unambiguous how to formulate the long-wave stability theory, using only the structure of the equations; $(b)$ embedding the stability problem in the two-wave interaction is a natural part of the multi-symplectic approach and provides further information about the nature of the instability; $(c)$ explicit results for weakly nonlinear water waves can be obtained; $(d)$ general conclusions about SCW instability for other systems are also deduced; $(e)$ it is straightforward to include meanflow effects. 
What is a SCW? The definition is implicit in the literature, and in Appendix A an explicit definition of SCWs is given. A solution, say $Z(x, y, t)$ of $(1.1)$, is called an SCW if it is periodic in $x, y$ and $t$, travelling in the $x$-direction (a function of $x$ and $t$ in linear combination only), and is invariant under reversibility in the $y$-direction. A definition of $y$-reversibility with examples is given in $\S 2$. An immediate and illuminating consequence of this definition is that SCWs have zero transverse momentum but certainly have non-zero longitudinal momentum. This property of SCWs is useful for interpreting the embedding of SCWs in a two-phase wavetrain: the embedding deforms the SCWs into waves with non-zero transverse momentum, thereby testing the susceptibility of SCWs to perturbation of the transverse momentum.

Much of the paper is devoted to the existence, properties and stability of the oblique two-wave interaction. Let $\eta(x, y, t)$ represent the free-surface elevation. The oblique two-wave interaction of water waves is a solution of the form

$$
\eta(x, y, t)=\widehat{\eta}\left(\theta_{1}, \theta_{2}\right)
$$

where

$$
\theta_{j}=k_{j} x+\ell_{j} y+\omega_{j} t+\theta_{j}^{0} \quad(j=1,2),
$$

and $\left(k_{j}, \ell_{j}\right)$ are the wavenumbers, $\omega_{j}$ are the frequencies, $\theta_{j}^{0}$ are phases, and $\hat{\eta}$ is a $2 \pi$ periodic function of $\theta_{1}$ and $\theta_{2}$. A short-crested wave is the special case: $k_{2}=k_{1}=k$, $\ell_{2}=-\ell_{1}=-\ell$ and $\omega_{2}=\omega_{1}=-\omega$, and it is reversible in the $y$-direction (invariant under change of sign of $y$; precise definition given in $\S 3$ and Appendix A). In the linear and weakly nonlinear limit this latter condition reduces to the familiar requirement of equal amplitudes of the two component waves.

The strategy is to construct variational principles for the general two-wave interaction. The variational principles provide natural Jacobians which contain information about the susceptibility of the SCW to distortion into oblique non-resonant two-wave interaction.

The linear stability problem for the general two-wave interaction is then formulated and a stability condition derived. Long-wave perturbations are of the form

$$
\eta(x, y, t)=\widehat{\eta}\left(\theta_{1}, \theta_{2}\right)+\operatorname{Re}\left(\mathscr{N}\left(\theta_{1}, \theta_{2}\right) \mathrm{e}^{\mathrm{i}(\alpha x+\beta y+\Omega t)}\right) \quad \text { with } \quad|\alpha|,|\beta| \ll 1 .
$$

The basic state is unstable when $\operatorname{Im}(\Omega)<0$. This condition can be strengthened to $\operatorname{Im}(\Omega) \neq 0$ by noting that the Hamiltonian symmetry assures us that there exists an eigenvalue with $\operatorname{Im}(\Omega)<0$ whenever one exists with $\operatorname{Im}(\Omega)>0$.

The main result is that all long-wave instabilities (of SCWs or the two-wave interaction) are predicted by the zeros of the quartic polynomial

$$
\Delta(\Omega, \alpha, \beta)=\operatorname{det}\left[\mathbf{N}_{2} \Omega^{2}+\mathbf{N}_{1} \Omega+\mathbf{N}_{0}\right]
$$

where $\boldsymbol{N}_{j}$ are $2 \times 2$ matrices dependent on $\alpha, \beta$ and the basic state. Precise expressions for these matrices are given in $\S 5$. Taking the limit to SCWs in the matrices $\boldsymbol{N}_{j}$ leads to a linear stability quartic for SCWs. Details of the results for SCWs are given in $\S \S 6$ and 7 .

The general result (i.e. not just for water waves) for the weakly nonlinear case can be summarized as follows. Let $D(\omega, k, \ell)$ be the dispersion relation for the linear problem, and suppose parameter values are chosen so that

$$
D_{\omega} \neq 0, \quad D_{k} \neq 0, \quad D_{\ell} \neq 0 .
$$

Consider a weakly nonlinear solution of (1.1) of the form

$$
Z(x, y, t)=\widehat{Z}(x, y, t)=A_{1} \xi \mathrm{e}^{\mathrm{i}(k x+\ell y-\omega t)}+A_{2} \xi \mathrm{e}^{\mathrm{i}(k x-\ell y-\omega t)}+\text { c.c. }+\cdots,
$$


where $\xi$ is an eigenvector determined by the linearized operator, $A_{1}$ and $A_{2}$ are complex amplitudes and the higher-order terms are higher order in $\left|A_{1}\right|$ and $\left|A_{2}\right|$. To leading order, the complex amplitudes satisfy

$$
\left.\begin{array}{l}
0=A_{1}\left(D(\omega, k, \ell)+a\left|A_{1}\right|^{2}+b\left|A_{2}\right|^{2}+\cdots\right) \\
0=A_{2}\left(D(\omega, k, \ell)+b\left|A_{1}\right|^{2}+a\left|A_{2}\right|^{2}+\cdots\right),
\end{array}\right\}
$$

where $a$ and $b$ are the coefficients for the nonlinear correction terms of the dispersion relation.

Clearly when $\left|A_{2}\right|=0$ and $\left|A_{1}\right| \neq 0$ (or vice versa) the basic state is a travelling wave with frequency change

$$
\omega=\omega_{0}+\omega_{2}^{T W}\left|A_{1}\right|^{2}+\cdots \quad \text { where } \quad \omega_{2}^{T W}=-\frac{a}{D_{\omega}},
$$

where $D\left(\omega_{0}, k, \ell\right)=0$ and $D_{\omega}$ is evaluated at $\omega=\omega_{0}$. On the other hand, SCWs satisfy $\left|A_{1}\right|=\left|A_{2}\right|$ and so their frequency change is

$$
\omega=\omega_{0}+\omega_{2}^{S C W}\left|A_{1}\right|^{2}+\cdots \quad \text { where } \quad \omega_{2}^{S C W}=-\frac{(a+b)}{D_{\omega}} .
$$

Now, add a long-wave perturbation to (1.5)

$$
Z(x, y, t)=\widehat{Z}(x, y, t)+\operatorname{Re}\left(\Xi \mathrm{e}^{\mathrm{i}(\alpha x+\beta y+\Omega t)}\right) \quad \text { with } \quad|\alpha|,|\beta| \ll 1 .
$$

For the linearization about weakly nonlinear SCWs, the stability quartic (1.4) for perturbations (1.9) has an interesting factorization into four branches (noting that $\left|A_{1}\right|=\left|A_{2}\right|:=|A|$ for these waves)

$$
\Omega=\left\{\begin{array}{l}
\frac{-D_{k} \alpha-D_{\ell} \beta}{D_{\omega}}-\sigma_{+}|A|+\cdots, \\
\frac{-D_{k} \alpha-D_{\ell} \beta}{D_{\omega}}+\sigma_{+}|A|+\cdots, \\
\frac{-D_{k} \alpha+D_{\ell} \beta}{D_{\omega}}-\sigma_{-}|A|+\cdots, \\
\frac{-D_{k} \alpha+D_{\ell} \beta}{D_{\omega}}+\sigma_{-}|A|+\cdots,
\end{array}\right.
$$

when $\beta \neq 0$ (transverse instability) with

$$
\left.\begin{array}{l}
\sigma_{+}^{2}=\left(\omega_{k k} \alpha^{2}+2 \omega_{k \ell} \alpha \beta+\omega_{\ell \ell} \beta^{2}\right) \omega_{2}^{T W}, \\
\sigma_{-}^{2}=\left(\omega_{k k} \alpha^{2}-2 \omega_{k \ell} \alpha \beta+\omega_{\ell \ell} \beta^{2}\right) \omega_{2}^{T W} .
\end{array}\right\}
$$

The derivatives of $\omega(k, \ell)$ are obtained by differentiating $D(\omega(k, \ell), k, \ell)=0$. Note that it is the TW correction and not the SCW correction to the frequency which appears at leading order in the stability exponents for transverse instability. 
(a)

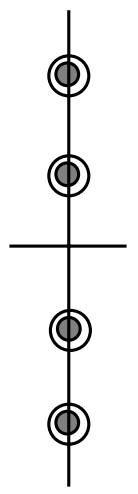

(b)

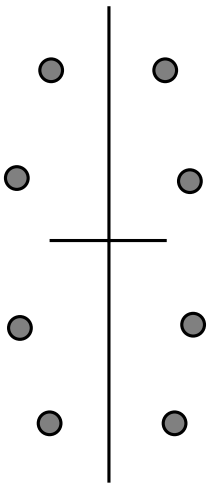

FIGURE 2. A schematic showing the typical qualitative position of the eigenvalues $\lambda= \pm \mathrm{i} \Omega$ for (1.10) when ( $a)|A|=0$ and $(b)|A|>0$.

When $\beta=0$ (longitudinal instability), the factorization changes to

$$
\Omega=\left\{\begin{array}{l}
-\frac{D_{k}}{D_{\omega}} \alpha-\mu_{+}|A|+\cdots, \\
-\frac{D_{k}}{D_{\omega}} \alpha+\mu_{+}|A|+\cdots, \\
-\frac{D_{k}}{D_{\omega}} \alpha-\mu_{-}|A|+\cdots, \\
-\frac{D_{k}}{D_{\omega}} \alpha+\mu_{-}|A|+\cdots,
\end{array}\right.
$$

with

$$
\left.\begin{array}{l}
\mu_{+}^{2}=\omega_{k k} \omega_{2}^{S C W} \alpha^{2}, \\
\mu_{-}^{2}=\omega_{k k}\left(2 \omega_{2}^{T W}-\omega_{2}^{S C W}\right) \alpha^{2} .
\end{array}\right\}
$$

A weakly nonlinear SCW is unstable if any of the four quantities $\sigma_{+}^{2}, \sigma_{-}^{2}, \mu_{+}^{2}$ or $\mu_{-}^{2}$ is negative.

Throughout it is assumed that $\sigma_{+}, \sigma_{-}, \mu_{+}$and $\mu_{-}$are non-vanishing and of order one. There are lines in perturbation wavenumber space, and particular values of the wavenumber vector of the SCWs, where these coefficients vanish. Equation (1.19) shows an example of these resonance lines, and further discussion is given in $\S 7$. When one of these coefficients vanishes, the weakly nonlinear stability properties are determined at the next order in $|A|$.

The stability exponents are $\lambda= \pm \mathrm{i} \Omega$ with $\Omega$ given by (1.10) or (1.12). When $|A|=0$ and $\beta \neq 0$ there is a double resonance, as shown schematically in figure $2(b)$, plotted in the complex $\lambda$-plane. The precise position of the resonances depends on the values of $(k, \ell)$ and $(\alpha, \beta)$. For $\beta=0$, both pairs coalesce and the resonance is fourfold. When $|A|>0$ the resonances split, and may become unstable. The most dramatic situation where $\beta \neq 0$ and two modes become unstable is shown in figure $2(b)$.

The information contained in (1.10) and (1.12) can be summarized as follows. Let $D(\omega, k, \ell)$ be the dispersion relation of the linearized problem and let $(\omega, k, \ell)$ be the frequency and wavenumbers of the weakly nonlinear SCW (or to leading order the values for the linearized problem). Then there are two alternatives for instability 
when $\beta \neq 0$. First, if $D_{\omega} \neq 0$ and

$$
\operatorname{det}\left[\begin{array}{cccc}
D_{\omega \omega} & D_{\omega k} & D_{\omega \ell} & D_{\omega} \\
D_{k \omega} & D_{k k} & D_{k \ell} & D_{k} \\
D_{\ell \omega} & D_{\ell k} & D_{\ell \ell} & D_{\ell} \\
D_{\omega} & D_{k} & D_{\ell} & 0
\end{array}\right]>0,
$$

the weakly nonlinear SCW is unstable. Secondly, if the determinant (1.14) is negative but

$$
\omega_{k k} \omega_{2}^{T W}<0,
$$

the basic SCW is unstable, where $\omega_{2}^{T W}$ is the frequency correction in (1.7), and $\omega_{k k}$ is the second derivative of $\omega$ associated with the $x$-direction only.

Longitudinal instabilities $(\beta=0)$ are determined by the signs of $\mu_{ \pm}^{2}$ in (1.13). The first condition,

$$
\omega_{k k} \omega_{2}^{S C W}<0,
$$

is similar to the condition proposed by Molloo-Christensen (1981), although $\omega_{k k}$ here depends on $\ell$ whereas in Mollo-Christensen (1981) the $\ell$-dependence is neglected (see discussion below). The second condition is

$$
\omega_{k k}\left(2 \omega_{2}^{T W}-\omega_{2}^{S C W}\right)<0 .
$$

This condition is related to the condition proposed by Roskes (1976b). Clearly a sufficient condition for longitudinal instability is $\mu_{+}^{2} \mu_{-}^{2}<0$ which results in

$$
0>\mu_{+}^{2} \mu_{-}^{2}=\omega_{k k}^{2} \alpha^{4} \omega_{2}^{S C W}\left(2 \omega_{2}^{T W}-\omega_{2}^{S C W}\right)=\frac{\omega_{k k}^{2}}{D_{\omega}^{2}} \alpha^{4}\left(a^{2}-b^{2}\right)
$$

or $|b|>|a|$ which is precisely the condition proposed by Roskes (1976b). However, this is only a sufficient condition. It misses the case where both $\mu_{-}^{2}$ and $\mu_{+}^{2}$ are negative, which occurs for water waves.

The condition (1.14) is a sufficient condition for the right-hand side in (1.11) to be factorizable with real factors. When $\sigma_{+}^{2}$ is factorizable, there is always a wedge emanating from the origin in the $(\alpha, \beta)$-plane where at least one of the roots of (1.10) is unstable. The determinant condition (1.14) is satisfied for all weakly nonlinear SCWs. However, the other potential transverse and longitudinal instabilities are worth investigating as they may produce more than one unstable mode, and unstable modes with higher growth rates.

Figure 3 shows a schematic of the position of the principal modes for SCWs when $\ell$ is small. In the small wedge around $\beta=0$ (the longitudinal instabilities) there is one pair of unstable modes; in the second wedge there are two unstable modes; in the third wedge this reduces to one unstable mode, and then for $\beta$ sufficiently large, there are no unstable modes. This figure changes for other $(k, \ell)$ values, and the other possible diagrams are shown in $\S 8$.

For water waves in deep water with gravity forces only, the dispersion relation is

$$
D(\omega, k, \ell)=\omega^{2}-g \nu, \quad v=\sqrt{k^{2}+\ell^{2}},
$$

and substitution into (1.14) shows that the determinant is always positive. It is immediate that there is a long-wave instability of weakly nonlinear short-crested water waves. There is, however, more information about the regions of instability contained 


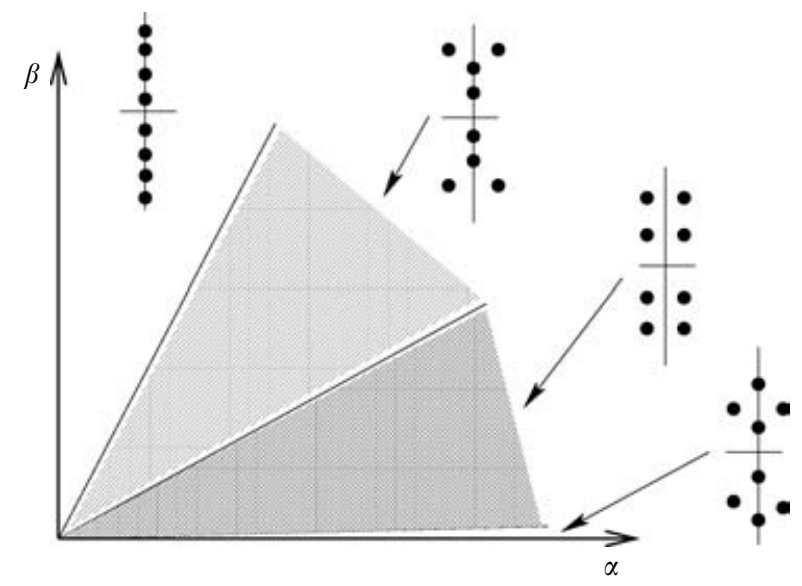

FIGURE 3. Position of unstable modes for weakly nonlinear gravity water waves (SCWs), in each wedge in the $(\alpha, \beta)$-plane when $2 \ell^{2}<k^{2}$.

in (1.11). Let

$$
s_{1}(k, \ell)=\frac{\sqrt{2} \ell-k}{\sqrt{2} k+\ell}, \quad s_{2}(k, \ell)=\frac{\sqrt{2} \ell+k}{\sqrt{2} k-\ell},
$$

then the coefficients (1.11) for the case of transverse instability of short-crested water waves can be factorized into

$$
\left.\begin{array}{l}
\sigma_{+}^{2}=-\frac{a}{8 v^{4}}\left(2 k^{2}-\ell^{2}\right)\left(\beta-s_{1} \alpha\right)\left(\beta-s_{2} \alpha\right), \\
\sigma_{-}^{2}=-\frac{a}{8 v^{4}}\left(2 k^{2}-\ell^{2}\right)\left(\beta+s_{1} \alpha\right)\left(\beta+s_{2} \alpha\right),
\end{array}\right\}
$$

where $a$ is as defined in (1.6). For deep-water waves, $a=-2 g v^{3}<0$. This factorization divides the $(\alpha, \beta)$-plane into wedges of stability and instability as shown schematically in figure 3. The results shown in figure 3 give a qualitative description of the transverse instability for weakly nonlinear short-crested Stokes waves when $k^{2}>2 \ell^{2}$.

The results in figure 3 agree with the numerical results of Ioualalen \& Kharif (1994) for small $|\alpha|+|\beta|$ : see figures 8 and 9 in Ioualalen \& Kharif (1994), where they are referred to as class Ia and class Ib instabilities. However, Ioualalen \& Kharif (1994) do not remark on the fact that two instabilities can occur at the same parameter values, but it appears to be implicit in their figures 8 and 9 .

A weakly nonlinear analysis of the stability of SCWs is given by Badulin et al. (1995) (hereinafter referred to as BSKI). Their results are predominantly qualitative, and therefore it is difficult to make explicit comparison. They also reduce the weakly nonlinear analysis to a quartic polynomial (see their (3.10)). However, they do not give explicit expressions for the coefficients and, more importantly, they do not find explicit expressions for the roots. In this paper, explicit expressions for the coefficients (see (7.10)), and explicit leading-order expressions for the roots are found.

The results presented here agree only partially with the earlier SCW stability results of Roskes (1976b) or Mollo-Christensen (1981). While these results have been criticized previously, we now have enough information to give a precise account of how these results are incorrect or incomplete.

In the paper of Mollo-Christensen (1981), it is proposed to use the Whitham criterion to predict longitudinal instability with respect to perturbations travelling 
in the same direction as the basic SCW. Using notation from this paper, MolloChristensen (1981) proposes that the SCW is unstable when

$$
\omega_{k k} \omega_{2}^{S C W}<0 .
$$

This condition agrees with the condition $\mu_{+}^{2}<0$ in (1.13), but it misses the condition $\mu_{-}^{2}<0$. The condition is missing because Mollo-Christensen assumes that SCW is a single-phase wavetrain rather than a two-phase wavetrain.

On the other hand, as first pointed out by Roberts (1983), there is an error in MolloChristensen (1981) in implementing the criterion (1.20). The derivative $\omega_{k k}$ with $\ell=0$ is used, resulting in $\omega_{k k}<0$ for all $\ell$. Hence the change of stability occurring when $\omega_{k k}$ changes sign is missed. See $\S 9$ for discussion of the sign of $\mu_{+}^{2}$. The condition (1.20) also misses the transverse instabilities of SCWs.

Roskes (1976b) proposes a system of coupled NLS equations as a model for the long-wave instability of SCWs,

$$
\left.\begin{array}{l}
\frac{\partial A_{1}}{\partial t}=\mathrm{i} \gamma_{1} \frac{\partial^{2} A_{1}}{\partial x^{2}}+\mathrm{i} A_{1}\left(p_{11}\left|A_{1}\right|^{2}+p_{12}\left|A_{2}\right|^{2}\right), \\
\frac{\partial A_{2}}{\partial t}=\mathrm{i} \gamma_{2} \frac{\partial^{2} A_{2}}{\partial x^{2}}+\mathrm{i} A_{2}\left(p_{21}\left|A_{1}\right|^{2}+p_{22}\left|A_{2}\right|^{2}\right) .
\end{array}\right\}
$$

In Roskes (1976b), $\beta$ is used instead of $p$. Notation is changed here to avoid confusion with the use of $\beta$ for the perturbation wavenumber.

The parameters are adjusted to represent SCWs: $\gamma_{1}=\gamma_{2}, p_{11}=p_{22}=p<0$, where $p$ is proportional to $a$ in (1.6), and $p_{12}=p_{21}$ is proportional to $b$ in (1.6). A basic state representative of an SCW is taken and then a linear stability analysis is given. Roskes (1976a) shows that in general such a state is unstable when

$$
\gamma_{1} \gamma_{2} \operatorname{det}\left[\begin{array}{ll}
p_{11} & p_{12} \\
p_{21} & p_{22}
\end{array}\right]<0
$$

Applied to (1.6) this condition states that SCWs are unstable when $\left|p_{12}\right|>|p|$ which is the condition stated in Roskes (1976b). This agrees with the sufficient condition (1.16), but misses the instability when both $\mu_{-}^{2}$ and $\mu_{+}^{2}$ are negative.

With (1.21) as a starting point, Roskes' analysis of this NLS system, as a representative model for longitudinal instability of SCWs, is correct. However, this coupled NLS model is not uniformly valid as a model equation for modulation of weakly nonlinear SCWs since it misses the transverse instabilities. This can be seen by looking at the derivation of this coupled NLS system in Roskes (1976a). The transformed slow space scale (denoted by $x$ here) is defined by

$$
x=\boldsymbol{u} \cdot X-\widetilde{\boldsymbol{c}_{g}} T
$$

in Roskes $(1976 a)$ where $\widetilde{\boldsymbol{c}_{g}}$ is the group velocity in the direction $\boldsymbol{u}$ where the group velocity of the two waves overlap, $X=\left(X_{1}, X_{2}\right)$ are slow space scales, and $T$ is a slow time scale. However, in order to balance the time derivative, Roskes introduces a new time scale $t=\varepsilon T$ (this $t$ is the variable in (1.12). Therefore, $x$ in (1.22) must be interpreted as

$$
x=\boldsymbol{u} \cdot X-\frac{1}{\varepsilon} \widetilde{\boldsymbol{c}_{g}} t .
$$

This expression shows that the scaling is not valid unless the group velocity overlap $\widetilde{\boldsymbol{c}_{g}}$ is of order $\varepsilon$. However, for SCWs of the water-wave problem it is of order unity. In the limit of long-crested waves the group velocities are nearly the same, but the 
weakly nonlinear expansion for SCWs can also be singular in this limit (Roberts \& Peregrine 1983).

The problem of deriving modulation equations for two-wave interaction when the group velocity overlap is of order one has been considered in detail by Knobloch \& Gibbon (1991) and Pierce \& Knobloch (1994). They show that in this case, the coupling terms change to non-local terms: one wave senses the other wave only through an average property of the other wave. The distinction is important as the stability results for modulation equations with non-local averaging differ significantly from the results for local equations such as (1.21). Pierce \& Knobloch (1994) derive the appropriate equations for modulation of standing waves, and it is reasonable to conjecture that the modulation equations for transverse instability of weakly nonlinear SCWs will be of a similar non-local form. The modulation equations derived by Pierce \& Knobloch (1994) for weakly nonlinear standing waves predict that the coupled wave is unstable if and only if the component travelling waves are unstable. Although the modulation equations of Pierce \& Knobloch (1994) do not apply to SCWs, if we extrapolate their results to SCWs, we find that they are consistent with the results found for transverse instability in this paper.

\section{Multi-symplectic structure of wave equations}

The theory for instability of short-crested waves is developed for the general class of PDEs (1.1). In this section, first a semilinear wave equation in two space dimensions will be used to illustrate the transformation to multi-symplectic form, and then the multi-symplectic formulation of water waves is recorded.

\subsection{Multi-symplectifying nonlinear wave equations}

Consider the class of semilinear wave equations,

$$
\frac{\partial^{2} u}{\partial t^{2}}-\frac{\partial^{2} u}{\partial x^{2}}-\frac{\partial^{2} u}{\partial y^{2}}+V^{\prime}(u)=0
$$

where $u(x, y, t)$ is scalar valued and $V(u)$ is a smooth nonlinear function with $V^{\prime \prime}(0)>0$. The canonical form of the Lagrangian is

$$
\mathscr{L}=\int \mathrm{L}\left(u, u_{t}, u_{x}\right) \mathrm{d} t \mathrm{~d} x \mathrm{~d} y, \quad \mathrm{~L}\left(u, u_{t}, u_{x}\right)=\frac{1}{2} u_{t}^{2}-\frac{1}{2} u_{x}^{2}-\frac{1}{2} u_{y}^{2}-V(u) .
$$

The canonical Hamiltonian formulation for the nonlinear wave equation is obtained by taking the Legendre transform with respect to time only, $v=\partial \mathrm{L} / \partial u_{t}=u_{t}$, and then the governing equations take the form

$$
\frac{\partial}{\partial t}\left(\begin{array}{l}
u \\
v
\end{array}\right)=\left[\begin{array}{rr}
0 & 1 \\
-1 & 0
\end{array}\right]\left(\begin{array}{c}
\frac{\delta \mathscr{H}}{\delta u} \\
\frac{\delta \mathscr{H}}{\delta v}
\end{array}\right), \mathscr{H}(u, v)=\int\left(\frac{1}{2} v^{2}+\frac{1}{2} u_{x}^{2}+\frac{1}{2} u_{y}^{2}+V(u)\right) \mathrm{d} x \mathrm{~d} y .
$$

Hamiltonian formulations of the nonlinear wave equation are widely used in analysis (see Kuksin 2000 and references therein). However a disadvantage of this formulation, when studying pattern formation, is that the Hamiltonian function and symplectic structure associated with (2.3) require definition of a space of functions over the $x$ and $y$-directions a priori. In the case of modulation instabilities, the basic state is periodic in space, but the perturbation class is in general quasi-periodic. 
Multi-symplecticity puts space and time on an equal footing. The governing equations are obtained by taking a Legendre transform with respect to all directions (a covariant or 'total' Legendre transform), $v=\partial \mathrm{L} / \partial u_{t}=u_{t}, w=\partial \mathrm{L} / \partial u_{x}=-u_{x}$ and $p=\partial \mathrm{L} / \partial u_{y}=-u_{y}$. The Legendre transform generates a new Hamiltonian functional

$$
S(u, v, w, p)=v u_{t}+w u_{x}+p u_{y}-\mathrm{L}=\frac{1}{2}\left(v^{2}-w^{2}-p^{2}\right)+V(u) .
$$

The new Lagrangian for the system is

$$
\mathscr{L}=\int \mathrm{L}(u, v, w, p) \mathrm{d} t \mathrm{~d} x \mathrm{~d} y, \quad \mathrm{~L}(u, v, w, p)=v u_{t}+w u_{x}+p u_{y}-S(u, v, w, p),
$$

and the governing equations are given by

$$
\begin{aligned}
& 0=\mathrm{L}_{u}=-v_{t}-w_{x}-p_{y}-S_{u}, \\
& 0=\mathrm{L}_{v}=u_{t}-S_{v}, \\
& 0=\mathrm{L}_{w}=u_{x}-S_{w}, \\
& 0=\mathrm{L}_{p}=u_{y}-S_{p},
\end{aligned}
$$

using standard fixed-endpoint conditions for the variations. However, these equations do not have a nice multi-symplectic structure, since the triple of symplectic operators are always degenerate. This structure is improved by observing that $v, w$ and $p$ satisfy the constraints $p_{x}-w_{y}=0, p_{t}+v_{y}=0$ and $v_{x}+w_{t}=0$. Therefore add these constraints to the Lagrangian (2.5) with Lagrange multipliers $\alpha_{1}, \alpha_{2}$ and $\alpha_{3}$. A divergence-free condition is imposed on the Lagrange multipliers: $\partial_{t} \alpha_{1}+\partial_{x} \alpha_{2}+\partial_{y} \alpha_{3}=0$. That this equation is the correct one is justified a posteriori: with this condition, the resulting multi-symplectic system provides an equivalent system of PDEs. With this additional constraint, the Lagrangian density is

$$
\begin{aligned}
\mathrm{L}\left(u, v, w, p, \alpha_{1}, \alpha_{2}, \alpha_{3}, \alpha_{4}\right)= & v u_{t}+w u_{x}+p u_{y}-S(u, v, w, p) \\
& +\alpha_{1}\left(p_{x}-w_{y}\right)+\alpha_{2}\left(p_{t}+v_{y}\right)-\alpha_{3}\left(v_{x}+w_{t}\right) \\
& +\alpha_{4}\left(\partial_{t} \alpha_{1}+\partial_{x} \alpha_{2}+\partial_{y} \alpha_{3}\right) .
\end{aligned}
$$

The governing equations are now

$$
\begin{gathered}
\mathbf{M} Z_{t}+\mathbf{K} Z_{x}+\mathbf{L} Z_{y}=\nabla S(Z) \quad Z \in \mathbb{R}^{8}, \\
\mathbf{M}=\left[\begin{array}{cccccccc}
0 & -1 & 0 & 0 & 0 & 0 & 0 & 0 \\
1 & 0 & 0 & 0 & 0 & 0 & 0 & 0 \\
0 & 0 & 0 & 0 & 0 & 0 & 1 & 0 \\
0 & 0 & 0 & 0 & 0 & -1 & 0 & 0 \\
0 & 0 & 0 & 0 & 0 & 0 & 0 & -1 \\
0 & 0 & 0 & 1 & 0 & 0 & 0 & 0 \\
0 & 0 & -1 & 0 & 0 & 0 & 0 & 0 \\
0 & 0 & 0 & 0 & 1 & 0 & 0 & 0
\end{array}\right], \quad \mathbf{K}=\left[\begin{array}{cccccccc}
0 & 0 & -1 & 0 & 0 & 0 & 0 & 0 \\
0 & 0 & 0 & 0 & 0 & 0 & 1 & 0 \\
1 & 0 & 0 & 0 & 0 & 0 & 0 & 0 \\
0 & 0 & 0 & 0 & -1 & 0 & 0 & 0 \\
0 & 0 & 0 & 1 & 0 & 0 & 0 & 0 \\
0 & 0 & 0 & 0 & 0 & 0 & 0 & -1 \\
0 & -1 & 0 & 0 & 0 & 0 & 0 & 0 \\
0 & 0 & 0 & 0 & 0 & 1 & 0 & 0
\end{array}\right],
\end{gathered}
$$


and

$$
\mathbf{L}=\left[\begin{array}{cccccccc}
0 & 0 & 0 & -1 & 0 & 0 & 0 & 0 \\
0 & 0 & 0 & 0 & 0 & -1 & 0 & 0 \\
0 & 0 & 0 & 0 & 1 & 0 & 0 & 0 \\
1 & 0 & 0 & 0 & 0 & 0 & 0 & 0 \\
0 & 0 & -1 & 0 & 0 & 0 & 0 & 0 \\
0 & 1 & 0 & 0 & 0 & 0 & 0 & 0 \\
0 & 0 & 0 & 0 & 0 & 0 & 0 & -1 \\
0 & 0 & 0 & 0 & 0 & 0 & 1 & 0
\end{array}\right], \quad Z=\left(\begin{array}{c}
u \\
v \\
w \\
p \\
\alpha_{1} \\
\alpha_{2} \\
\alpha_{3} \\
\alpha_{3}
\end{array}\right),
$$

The formulation (2.7) is remarkable in that all three of the operators $\mathbf{M}, \mathbf{K}$ and $\mathbf{L}$ are non-degenerate, and so they each define canonical symplectic structures on $\mathbb{R}^{8}$.

A fundamental property of the scalar nonlinear wave equation (2.1), that is important for the existence of short-crested waves, is reversibility in $y$. If $u(x, y, t)$ is a solution of (2.1), then clearly $u(x,-y, t)$ is also a solution. In the multi-symplectification of (2.1), this reversibility is defined by the action

$$
\mathscr{R} Z(x, y, t):=\mathbf{R} Z(x,-y, t) \quad \text { with } \quad \mathbf{R}=\operatorname{diag}(1,1,1,-1,-1,-1,1,-1) .
$$

The matrix $\mathbf{R}$ is an involution (has the property $\mathbf{R}^{2}=\mathbf{I}$ ) and satisfies

$$
\mathbf{R M}=\mathbf{M R}, \quad \mathbf{R K}=\mathbf{K R}, \quad \mathbf{R L}=-\mathbf{L R}, \quad S(\mathscr{R} Z)=S(Z) .
$$

In turn, the properties (2.9) imply that $\mathscr{R} Z$ is a solution of the wave equation in the form (2.7) whenever $Z$ is a solution. The nonlinear wave equation (2.1) is reversible in $x$ and $t$ as well, and a multi-symplectic $t$-reversor and $x$-reversor can also be defined, but they are not required in the general theory for short crested waves.

In addition to being a simple example, the nonlinear wave equation has an interesting property which is quite different from water waves: the determinant condition (1.14) is not violated. The dispersion relation for (2.1) linearized about the trivial state $u=0$ is

$$
D(\omega, k, \ell)=\omega^{2}-k^{2}-\ell^{2}-V^{\prime \prime}(0),
$$

where $V^{\prime \prime}(0)>0$ by hypothesis. Hence,

$$
\operatorname{det}\left[\begin{array}{cccc}
D_{\omega \omega} & D_{\omega k} & D_{\omega \ell} & D_{\omega} \\
D_{k \omega} & D_{k k} & D_{k \ell} & D_{k} \\
D_{\ell \omega} & D_{\ell k} & D_{\ell \ell} & D_{\ell} \\
D_{\omega} & D_{k} & D_{\ell} & 0
\end{array}\right]=\operatorname{det}\left[\begin{array}{cccc}
2 & 0 & 0 & 2 \omega \\
0 & -2 & 0 & -2 k \\
0 & 0 & -2 & -2 \ell \\
2 \omega & -2 k & -2 \ell & 0
\end{array}\right]=-16 V^{\prime \prime}(0)<0
$$

Therefore, by choosing $V^{\prime \prime \prime}(0)=0$ and $V^{\prime \prime \prime \prime}(0)>0\left(\operatorname{since} \operatorname{sign}\left(\omega_{k k} \omega_{2}^{T W}\right)=\operatorname{sign}\left(V^{\prime \prime \prime \prime}(0)\right)\right.$ in this case), the weakly nonlinear SCWs of (2.1) are stable to long-wave transverse perturbations. They may, of course, still be unstable to short-wave transverse perturbations or longitudinal perturbations.

\subsection{Multi-symplectic structure of water waves}

The multi-symplectic formulation of water waves of Bridges $(1996,1997 a)$ is used, and the details required are recorded here. Restrict attention to inviscid irrotational water waves of constant density on an infinite depth fluid.

Let $(x, y) \in \mathbb{R}^{2}$ denote the horizontal coordinates and $z$ the vertical coordinate. Denote by $\phi(x, y, z, t)$ the velocity potential. The fluid is bounded above by the surface $z=\eta(x, y, t)$. In the interior of the fluid, the velocity potential satisfies Laplace's 
equation

$$
\Delta \phi=\phi_{x x}+\phi_{y y}+\phi_{z z}=0 \text { for }-\infty<z<\eta(x, y, t)
$$

and is quiescent far from the surface

$$
\nabla \phi \rightarrow 0 \quad \text { as } \quad z \rightarrow-\infty .
$$

At the free surface, the functions $(\phi, \eta)$ satisfy the kinematic and dynamic boundary conditions

$$
\left.\begin{array}{l}
\eta_{t}+\phi_{x} \eta_{x}+\phi_{y} \eta_{y}-\phi_{z}=0 \\
\phi_{t}+\frac{1}{2}\left(\phi_{x}^{2}+\phi_{y}^{2}+\phi_{z}^{2}\right)+g \eta=0
\end{array}\right\} \quad \text { at } z=\eta(x, y, t)
$$

where $g$ is the gravitational constant.

To multi-symplectify, introduce new variables $Z=(\Phi, \eta, \phi, u, v)$ where

$$
\Phi=\left.\phi\right|_{z=\eta}, \quad u=\phi_{x}, \quad v=\phi_{y}, \quad \boldsymbol{u}=\left.u\right|_{z=\eta}, \quad \boldsymbol{v}=\left.v\right|_{z=\eta} .
$$

The functions $(\Phi, \eta)$ are, for each $(x, y, t)$, real numbers whereas $(\phi, u, v)$ are dependent also on the cross-section $z \in(-\infty, \eta)$. Using the fact that

$$
\Phi_{t}=\left.\left[\phi_{t}+\phi_{z} \eta_{t}\right]\right|_{z=\eta},
$$

with similar relations for $\Phi_{x}$ and $\Phi_{y}$, and the kinematic condition, leads to the identity

$$
\Phi_{t}+\boldsymbol{u} \Phi_{x}+\boldsymbol{v} \Phi_{y}=\left.\left[\phi_{t}+\left(\phi_{x}^{2}+\phi_{y}^{2}+\phi_{z}^{2}\right)\right]\right|_{z=\eta}
$$

With these coordinates, the governing equations can be written in the form

$$
\mathbf{M}(Z) Z_{t}+\mathbf{K}(Z) Z_{x}+\mathbf{L}(Z) Z_{y}=\nabla S(Z)
$$

with

$$
S(Z)=\frac{1}{2} \int_{-\infty}^{\eta}\left(u^{2}+v^{2}-\phi_{z}^{2}\right) \mathrm{d} z-\frac{1}{2} g \eta^{2},
$$

and the associated side conditions on elements of $Z$,

$$
\left.\phi\right|_{z=\eta}=\Phi, \quad|\nabla \phi| \rightarrow 0 \quad \text { as } z \rightarrow-\infty .
$$

The operators $\mathbf{M}(Z), \mathbf{K}(Z)$ and $\mathbf{L}(Z)$ are defined by

$$
\mathbf{M}(Z)=\left(\begin{array}{ccccc}
0 & -1 & 0 & 0 & 0 \\
1 & 0 & 0 & 0 & 0 \\
0 & 0 & 0 & 0 & 0 \\
0 & 0 & 0 & 0 & 0 \\
0 & 0 & 0 & 0 & 0
\end{array}\right), \quad \mathbf{K}(Z)=\left(\begin{array}{ccccc}
0 & -\boldsymbol{u} & 0 & 0 & 0 \\
\boldsymbol{u} & 0 & 0 & 0 & 0 \\
0 & 0 & 0 & -1 & 0 \\
0 & 0 & 1 & 0 & 0 \\
0 & 0 & 0 & 0 & 0
\end{array}\right)
$$

and

$$
\mathbf{L}(Z)=\left(\begin{array}{ccccc}
0 & -\boldsymbol{v} & 0 & 0 & 0 \\
\boldsymbol{v} & 0 & 0 & 0 & 0 \\
0 & 0 & 0 & 0 & -1 \\
0 & 0 & 0 & 0 & 0 \\
0 & 0 & 1 & 0 & 0
\end{array}\right)
$$

To verify that the right-hand side of $(2.15)$ is the gradient of some functional $S$, we first introduce a suitable inner product. For vector-valued functions of the type $Z$, where the first two components are scalar-valued and the last three components are 
defined on the cross-section $z \in(-\infty, \eta)$, we use the following inner product

$$
\langle U, V\rangle_{\eta}=U_{1} V_{1}+U_{2} V_{2}+\int_{-\infty}^{\eta}\left(U_{3} V_{3}+U_{4} V_{4}+U_{5} V_{5}\right) \mathrm{d} z
$$

Note that the inner product is dependent on $\eta$, and this is indicated by the subscript on the inner product. The gradient of $S(Z),(2.16)$, with respect to the inner product, $(2.20)$, is

$$
\nabla S(Z) \stackrel{\text { def }}{=}\left(\begin{array}{c}
\delta S / \delta \Phi \\
\delta S / \delta \eta \\
\delta S / \delta \phi \\
\delta S / \delta u \\
\delta S / \delta v
\end{array}\right)=\left(\begin{array}{c}
-\left.\phi_{z}\right|_{z=\eta} \\
\left.\frac{1}{2}\left(\boldsymbol{u}^{2}+\boldsymbol{v}^{2}+\phi_{z}^{2}\right)\right|_{z=\eta}-g \eta \\
\phi_{z z} \\
u \\
v
\end{array}\right) .
$$

The water-wave problem has the appropriate $y$-reversibility that is required for the existence of SCWs. Let

$$
\mathbf{R}=\operatorname{diag}(1,1, \mathbf{I}, \mathbf{I},-\mathbf{I}) .
$$

Then it is easily verified that

$$
\mathbf{R M}(\mathscr{R} Z)=+\mathbf{M}(Z) \mathbf{R}, \quad \mathbf{R L}(\mathscr{R} Z)=+\mathbf{K}(Z) \mathbf{R}, \quad \mathbf{R L}(\mathscr{R} Z)=-\mathbf{L}(Z) \mathbf{R},
$$

and $S(\mathscr{R} Z)=S(Z)$, where $\mathscr{R} Z=\mathbf{R} Z(x,-y, t)$.

The skew-symmetric operators $\mathbf{M}(Z), \mathbf{K}(Z)$ and $\mathbf{L}(Z)$ are non-constant. However, with a simple transformation, they can be reduced to constant skew-symmetric operators (see Bridges 2001). Therefore, it will be assumed hereinafter that the waterwave equations are transformed and so are in the standard form (1.1).

The multi-symplectic formulation of water waves is a generalization of the classical Hamiltonian formulation of water waves due to Zakharov (1968). Defining,

$$
\widetilde{\nabla} H(Z)=\nabla S(Z)-\mathbf{K}(Z) Z_{x}-\mathbf{L}(Z) Z_{y}
$$

where $\widetilde{\nabla}$ is a gradient operator defined with respect to an inner product that includes integration over $x$ and $y$, the equations can be written in the form

$$
\mathbf{M}(Z) Z_{t}=\widetilde{\nabla} H(Z) .
$$

This system is the Zakharov formulation rewritten in terms of the $Z$-variables. The multi-symplectic structure provides a refinement of the classical Hamiltonian structure, in that it decomposes the Hamiltonian to generate independent symplectic structures for the $x$ - and $y$-directions.

\section{The general oblique two-wave interaction}

Motivated by the nonlinear wave equation and the water-wave problem, the starting point for the analysis is the general class of abstract Hamiltonian PDEs of the form

$$
\mathbf{M} Z_{t}+\mathbf{K} Z_{x}+\mathbf{L} Z_{y}=\nabla S(Z), \quad Z \in \mathbb{H},
$$

under the hypotheses that $\mathbf{M}, \mathbf{K}$ and $\mathbf{L}$ are any constant skew-symmetric operators, $S$ is any given smooth function, which does not depend explicitly on $x, y$ or $t$. The linear space $\mathbb{H}$ is either $\mathbb{R}^{n}$ or in the case of water waves it is an inner product space of functions in the $z$-direction. The precise specification of $\mathbb{H}$ is not required in the sequel. On $\mathbb{H}$, the standard inner product will be denoted by $\langle\cdot, \cdot\rangle$. 
It is also assumed that there is a reversibility in $y$ with a multi-symplectic action of the reversor:

$$
\mathscr{R} Z(x, y, t)=\mathbf{R} Z(x,-y, t),
$$

for some linear operator $\mathbf{R}: \mathbb{H} \rightarrow \mathbb{H}$ which is involutive (i.e. $\mathbf{R}^{2}=\mathbf{I}$ ) and preserves the inner product, and satisfies (2.9) for (3.1). In this setting, an abstract definition of a short-crested wave can be given (see Appendix A).

In this section, the general two-wave interaction is considered; that is, general solutions of (3.1) of the form

$$
Z(x, y, t)=\widehat{Z}\left(\theta_{1}, \theta_{2}\right) \quad \text { where } \quad \theta_{j}=k_{j} x+\ell_{j} y+\omega_{j} t \quad(j=1,2),
$$

and $\widehat{Z}$ is a $2 \pi$-periodic function of $\theta_{1}$ and $\theta_{2}$. There is an arbitrary phase shift in each $\theta_{j}$ which is suppressed for brevity.

In addition to its importance as an embedding for SCWs, the oblique two-wave interaction has independent interest in remote-sensing stochastic models, and a model for the double-peaked power spectrum observed in shallow water-wave dynamics Longuet-Higgins 1962; Willebrand 1975; Weber \& Barrick 1977; Pierson 1993; Elfouhaily et al. 2000 and references therein).

The main result of this section is a constrained variational principle for the twowave interaction which generalizes previous variational principles for quasi-periodic patterns (Bridges 1998) and collinear two-phase wavetrains (Bridges \& Laine-Pearson 2001). Variational principles can be derived for SCWs directly, as in Bridges, Dias \& Menasce (2001) for example, but these variational principles for SCWs do not contain enough information for a stability analysis.

The solutions $\widehat{Z}\left(\theta_{1}, \theta_{2}\right)$ can also be interpreted as steady waves travelling in some oblique direction (Milewski \& Keller 1996). Let

$$
\Theta_{1}=\frac{\ell_{2} \theta_{1}-\ell_{1} \theta_{2}}{k_{1} \ell_{2}-k_{2} \ell_{1}}, \quad \Theta_{2}=\frac{k_{2} \theta_{1}-k_{1} \theta_{2}}{k_{1} \ell_{2}-k_{2} \ell_{1}} .
$$

Then clearly,

$$
\Theta_{1}=x-c_{x} t, \quad \Theta_{2}=y-c_{y} t, \quad \text { with } \quad\left(c_{x}, c_{y}\right)=\left(\frac{\omega_{2} \ell_{1}-\omega_{1} \ell_{2}}{k_{1} \ell_{2}-\ell_{1} k_{2}}, \frac{\omega_{2} k_{1}-\omega_{1} k_{2}}{k_{1} \ell_{2}-\ell_{1} k_{2}}\right) .
$$

The transformation $\left(\theta_{1}, \theta_{2}\right) \mapsto\left(\Theta_{1}, \Theta_{2}\right)$ is invertible if $k_{1} \ell_{2}-k_{2} \ell_{1} \neq 0$. Note that this non-degeneracy condition holds even in the SCW limit (reducing to $k \ell \neq 0$ ). In transformed coordinates, a doubly periodic wave can be expressed in the form

$$
\widehat{Z}\left(\theta_{1}, \theta_{2}\right)=\widehat{W}\left(\Theta_{1}, \Theta_{2}\right)=\widehat{W}\left(x-c_{x} t, y-c_{y} t\right),
$$

i.e. a steady wave travelling with phasespeed vector $c=\left(c_{x}, c_{y}\right)$. However, for the variational characterization, the primitive form of $\widehat{Z}\left(\theta_{1}, \theta_{2}\right)$ is used as the parameter structure is more useful.

The extension from SCWs to the non-resonant two-wave interaction takes a resonant wave to a non-resonant wave, and therefore we would expect small divisors. However, it is not this embedding that gives rise to small divisors, because there is a continuous symmetry (the translation invariance in the $y$-direction on periodic functions gives an $O(2)$ symmetry), and so the variation of the frequencies and wavenumbers is smooth. In finite dimensions this is reminiscent of the spherical pendulum, and in infinite dimensions it is reminiscent of the similar issues with standing waves and their embedding in a collinear two-wave interaction (see Bridges \& Laine-Pearson 2004 for further discussion of this issue). 
On the other hand, there is an intrinsic issue of small divisors that arises owing to the countable number of pure imaginary eigenvalues in the spectrum of the linearization about the trivial state. See Craig \& Nicholls (2002, $§ 4.4)$, for a discussion of this issue for three-dimensional water waves. However, when we consider capillary-gravity three-dimensional waves instead of pure gravity waves three-dimensional waves, the small-divisor issue disappears and a rigorous proof of such doubly periodic waves can be obtained (Craig \& Nicholls 2000).

The governing equation for a general two-wave interaction $\widehat{Z}\left(\theta_{1}, \theta_{2}\right)$ is obtained from (3.1) which transforms to

$$
\mathbf{J}_{1} \frac{\partial \widehat{Z}}{\partial \theta_{1}}+\mathbf{J}_{2} \frac{\partial \widehat{Z}}{\partial \theta_{2}}=\nabla S(\widehat{Z}) \quad \text { where } \quad \mathbf{J}_{j}=\omega_{j} \mathbf{M}+k_{j} \mathbf{K}+\ell_{j} \mathbf{L} .
$$

The operators $\mathbf{J}_{j} \partial_{\theta_{j}}$ are formally gradient operators, and this is the basis of a variational principle. The product of the skew-symmetric operator $\mathbf{J}_{j}$ and the derivative $\partial_{\theta_{j}}$ is symmetric. Therefore the product can define a quadratic form whose gradient then formally recovers the operator.

For $j=1,2$, define the following six functionals for the two interacting waves

$$
\left.\begin{array}{l}
\mathscr{A}_{j}(Z)=\oint \frac{1}{2}\left\langle\mathbf{M} \frac{\partial Z}{\partial \theta_{j}}, Z\right\rangle \mathrm{d} \theta, \quad \mathscr{B}_{j}(Z)=\oint \frac{1}{2}\left\langle\mathbf{K} \frac{\partial Z}{\partial \theta_{j}}, Z\right\rangle \mathrm{d} \theta, \\
\mathscr{C}_{j}(Z)=\oint \frac{1}{2}\left\langle\mathbf{L} \frac{\partial Z}{\partial \theta_{j}}, Z\right\rangle \mathrm{d} \theta \quad \text { where } \quad \oint() \mathrm{d} \theta=\frac{1}{(2 \pi)^{2}} \int_{0}^{2 \pi} \int_{0}^{2 \pi}() \mathrm{d} \theta_{1} \mathrm{~d} \theta_{2} .
\end{array}\right\}
$$

For the case of water waves, these functionals are expressible in the classical form

$$
\mathscr{A}_{j}(Z)=\oint-\Phi \frac{\partial \eta}{\partial \theta_{j}} \mathrm{~d} \theta, \quad \mathscr{B}_{j}(Z)=\oint \int_{-\infty}^{\eta} u \frac{\partial \phi}{\partial \theta_{j}} \mathrm{~d} z \mathrm{~d} \theta, \quad \mathscr{C}_{j}(Z)=\oint \int_{-\infty}^{\eta} v \frac{\partial \phi}{\partial \theta_{j}} \mathrm{~d} z \mathrm{~d} \theta .
$$

The functionals $\mathscr{A}_{j}$ can be identified with a multi-phase form of wave action and the functionals $\mathscr{B}_{j}$ and $\mathscr{C}_{j}$ can be identified with wave action fluxes (Whitham 1974). The difference here is that we do not use a Lagrangian formulation, and the actions and action fluxes have a geometrical characterization (Bridges 1997b). With the geometrical formulation, the actions and action fluxes enter the linear stability analysis in an explicit way and so stability results can be obtained without having to use a modulation equation such as the multi-phase modulation equation of Ablowitz \& Benney (1970).

Consider the Lagrange functional

$$
\mathscr{F}(Z ; \omega, k, \ell)=\mathscr{S}(Z)-\sum_{j=1}^{2}\left(\omega_{j} \mathscr{A}_{j}+k_{j} \mathscr{B}_{j}+\ell_{j} \mathscr{C}_{j}\right) \quad \text { where } \quad \mathscr{S}(Z)=\oint S(Z) \mathrm{d} \theta .
$$

Then using an inner product that includes integration over $\theta$, the first variation of $\mathscr{F}$ is the governing equation (3.4).

This functional leads to the following constrained variational principle. Let

$$
\mathfrak{C}(Z)=\left\{Z: \mathscr{A}_{j}(Z)=I_{j}, \mathscr{B}_{j}(Z)=I_{2+j}, \mathscr{C}_{j}(Z)=I_{4+j}, \quad j=1,2, \quad I \in \mathbb{R}^{6}\right\},
$$

where $I=\left(I_{1}, \ldots, I_{6}\right)$ are assigned level sets of the functionals. Then a two-wave interaction solution $\widehat{Z}\left(\theta_{1}, \theta_{2}\right)$ can be characterized as a critical point of $\mathscr{S}$ with $\widehat{Z}$ restricted to the set $\mathfrak{C}$. The Lagrange necessary condition is $\nabla \mathscr{F}=0$ (3.4). 
There are two immediate consequences of the Lagrange multiplier theory. First, the six parameters $\left(\omega_{j}, k_{j}, \ell_{j}\right) j=1,2$ are Lagrange multipliers and therefore satisfy

$$
\omega_{1}=\frac{\partial \mathscr{S}}{\partial I_{1}}, \quad k_{1}=\frac{\partial \mathscr{S}}{\partial I_{2}} \quad \ell_{1}=\frac{\partial \mathscr{S}}{\partial I_{3}} \quad \omega_{2}=\frac{\partial \mathscr{S}}{\partial I_{4}}, \quad k_{2}=\frac{\partial \mathscr{S}}{\partial I_{5}}, \quad \ell_{2}=\frac{\partial \mathscr{S}}{\partial I_{6}} .
$$

Secondly, the constrained variational principle is non-degenerate if

$$
\operatorname{det}\left[\begin{array}{llllll}
\frac{\partial \omega_{1}}{\partial I_{1}} & \frac{\partial \omega_{1}}{\partial I_{2}} & \frac{\partial \omega_{1}}{\partial I_{3}} & \frac{\partial \omega_{1}}{\partial I_{4}} & \frac{\partial \omega_{1}}{\partial I_{5}} & \frac{\partial \omega_{1}}{\partial I_{6}} \\
\frac{\partial k_{1}}{\partial I_{1}} & \frac{\partial k_{1}}{\partial I_{2}} & \frac{\partial k_{1}}{\partial I_{3}} & \frac{\partial k_{1}}{\partial I_{4}} & \frac{\partial k_{1}}{\partial I_{5}} & \frac{\partial k_{1}}{\partial I_{6}} \\
\frac{\partial \ell_{1}}{\partial I_{1}} & \frac{\partial \ell_{1}}{\partial I_{2}} & \frac{\partial \ell_{1}}{\partial I_{3}} & \frac{\partial \ell_{1}}{\partial I_{4}} & \frac{\partial \ell_{1}}{\partial I_{5}} & \frac{\partial \ell_{1}}{\partial I_{6}} \\
\frac{\partial \omega_{2}}{\partial I_{1}} & \frac{\partial \omega_{2}}{\partial I_{2}} & \frac{\partial \omega_{2}}{\partial I_{3}} & \frac{\partial \omega_{2}}{\partial I_{4}} & \frac{\partial \omega_{2}}{\partial I_{5}} & \frac{\partial \omega_{2}}{\partial I_{6}} \\
\frac{\partial k_{2}}{\partial I_{1}} & \frac{\partial k_{2}}{\partial I_{2}} & \frac{\partial k_{2}}{\partial I_{3}} & \frac{\partial k_{2}}{\partial I_{4}} & \frac{\partial k_{2}}{\partial I_{5}} & \frac{\partial k_{2}}{\partial I_{6}} \\
\frac{\partial \ell_{2}}{\partial I_{1}} & \frac{\partial \ell_{2}}{\partial I_{2}} & \frac{\partial \ell_{2}}{\partial I_{3}} & \frac{\partial \ell_{2}}{\partial I_{4}} & \frac{\partial \ell_{2}}{\partial I_{5}} & \frac{\partial \ell_{2}}{\partial I_{6}}
\end{array}\right] \neq 0 .
$$

Using (3.8), the condition (3.9) is equivalent to the non-degeneracy of the Hessian of $\mathscr{S}$ with respect to $I_{1}, \ldots, I_{6}$. A condition which is equivalent to (3.9) is

$$
\operatorname{det}\left[\begin{array}{ccc}
\frac{\delta \mathscr{A}}{\delta \omega} & \frac{\delta \mathscr{A}}{\delta k} & \frac{\delta \mathscr{A}}{\delta l} \\
\frac{\delta \mathscr{B}}{\delta \omega} & \frac{\delta \mathscr{B}}{\delta k} & \frac{\delta \mathscr{B}}{\delta l} \\
\frac{\delta \mathscr{C}}{\delta \omega} & \frac{\delta \mathscr{C}}{\delta k} & \frac{\delta \mathscr{C}}{\delta \ell}
\end{array}\right] \neq 0,
$$

where

$$
\frac{\delta \mathscr{A}}{\delta \omega}=\left(\begin{array}{cc}
\frac{\partial \mathscr{A}_{1}}{\partial \omega_{1}} & \frac{\partial \mathscr{A}_{1}}{\partial \omega_{2}} \\
\frac{\partial \mathscr{A}_{2}}{\partial \omega_{1}} & \frac{\partial \mathscr{A}_{2}}{\partial \omega_{2}}
\end{array}\right), \quad \frac{\delta \mathscr{A}}{\delta k}=\left(\begin{array}{cc}
\frac{\partial \mathscr{A}_{1}}{\partial k_{1}} & \frac{\partial \mathscr{A}_{1}}{\partial k_{2}} \\
\frac{\partial \mathscr{A}_{2}}{\partial k_{1}} & \frac{\partial \mathscr{A}_{2}}{\partial k_{2}}
\end{array}\right), \quad \frac{\delta \mathscr{A}}{\delta \ell}=\left(\begin{array}{cc}
\frac{\partial \mathscr{A}_{1}}{\partial \ell_{1}} & \frac{\partial \mathscr{A}_{1}}{\partial \ell_{2}} \\
\frac{\partial \mathscr{A}_{2}}{\partial \ell_{1}} & \frac{\partial \mathscr{A}_{2}}{\partial \ell_{2}}
\end{array}\right),
$$

with analogous $2 \times 2$ matrices for $\mathscr{B}$ and $\mathscr{C}$.

It follows from the variational principle that the matrices in (3.9) and (3.10) are symmetric. Hence

$$
\frac{\delta \mathscr{B}}{\delta \omega}=\frac{\delta \mathscr{A}^{T}}{\delta k}, \quad \frac{\delta \mathscr{C}}{\delta \omega}=\frac{\delta \mathscr{A}^{T}}{\delta l}, \quad \frac{\delta \mathscr{C}}{\delta k}=\frac{\delta \mathscr{B}^{T}}{\delta l} .
$$

Although we have restricted attention to the two-wave interaction here, it should be apparent that the basic formulation can be generalized to $N$-wave interactions. When there are $N$ interacting waves, there will be $3 N$ functionals and $3 N$ Lagrange multipliers. For the case of the three-wave interaction of water waves, this variational principle has been applied by Laine-Pearson (2002) to obtain results for the weakly nonlinear three-wave interaction.

\section{CAmbridge JDUANALS}




\section{Weakly nonlinear oblique two-wave interaction of water waves}

In this section, the variational principle of $\S 3$ is applied to weakly nonlinear water waves. The motivation is twofold: to derive existing results in the literature on the twowave interaction (e.g. Longuet-Higgins 1962; Weber \& Barrick 1977; Pierson 1993) from a variational perspective, and secondly, to obtain information which is used for the limit to SCWs. Some generalities about the weakly nonlinear two-wave interaction are also discussed.

At the linear level, a two-wave interaction solution of (3.1) is of the form

$$
\widehat{Z}\left(\theta_{1}, \theta_{2}\right)=\sum_{j=1}^{2}\left(A_{j} \xi_{j} \exp \left(\mathrm{i} \theta_{j}\right)+\text { c.c. }\right) \quad \text { with } \quad D\left(\omega_{j}, k_{j}, \ell_{j}\right)=0, \quad j=1,2,
$$

for any complex numbers $A_{1}$ and $A_{2}$, where $\xi_{j}$ is an eigenvector and $D(\omega, k, \ell)$ is the dispersion function. For gravity water waves in infinite depth, $D(\omega, k, \ell)=\omega^{2}-$ $g \sqrt{k^{2}+\ell^{2}}$.

The simplest nonlinear problem of pairwise interaction is then to study the persistence of such a wave interaction in the nonlinear problem for small amplitude. Such an interaction will not persist for all $\left(A_{1}, A_{2}\right) \in \mathbb{C}^{2}$ and one purpose of a weakly nonlinear analysis is to determine under what conditions we can expect such an interaction to persist. The weakly nonlinear theory leads to a set of amplitude equations of the form

$$
\left.\begin{array}{l}
0=A_{1}\left(D\left(\omega_{1}, k_{1}, \ell_{1}\right)+\Lambda_{11}\left|A_{1}\right|^{2}+\Lambda_{12}\left|A_{2}\right|^{2}+\cdots\right) \\
0=A_{2}\left(D\left(\omega_{2}, k_{2}, \ell_{2}\right)+\Lambda_{21}\left|A_{1}\right|^{2}+\Lambda_{22}\left|A_{2}\right|^{2}+\cdots\right)
\end{array}\right\}
$$

with $\Lambda_{12}=\Lambda_{21}$. These equations generalize the amplitude equations (1.6) for SCWs to amplitude equations for the two-wave interaction. Here we give a brief account of the derivation of this equation for weakly nonlinear two-wave interaction for water waves.

According to the variational principle, the solutions correspond to critical points of $\mathscr{S}$ restricted to level sets of the functionals $\left(\mathscr{A}_{j}, \mathscr{B}_{j}, \mathscr{C}_{j}\right)$ for $j=1,2$. The necessary condition for the variational principle is to find critical points of the unconstrained functional (3.7). We seek solutions that are $2 \pi$-periodic in $\theta_{1}$ and $\theta_{2}$ through a double Fourier series of the form

$$
\widehat{Z}\left(\theta_{1}, \theta_{2}\right)=\sum_{(m, n) \in \mathbb{Z}^{2}} Z_{m n} \exp \left(\mathrm{i}\left(m \theta_{1}+n \theta_{2}\right)\right) .
$$

Since $Z=(\Phi, \eta, \phi, u, v)^{T}$ we can determine $\Phi, u$ and $v$ from $\phi$ and $\eta$ using

$$
\Phi=\left.\phi\right|_{z=\eta}, \quad\left(\begin{array}{l}
u \\
v
\end{array}\right)=\left(\begin{array}{ll}
k_{1} & k_{2} \\
\ell_{1} & \ell_{2}
\end{array}\right)\left(\begin{array}{l}
\partial \phi / \partial \theta_{1} \\
\partial \phi / \partial \theta_{2}
\end{array}\right) .
$$

The problem is then reduced to solving for the velocity potential and free-surface elevation. A leading-order expansion for them is

$$
\begin{aligned}
\eta\left(\theta_{1}, \theta_{2}\right)= & A_{1} \mathrm{e}^{\mathrm{i} \theta_{1}}+A_{2} \mathrm{e}^{\mathrm{i} \theta_{2}}+a_{21}+a_{22} \mathrm{e}^{2 \mathrm{i} \theta_{1}}+a_{23} \mathrm{e}^{2 \mathrm{i} \theta_{2}} \\
& +a_{24} \mathrm{e}^{\mathrm{i}\left(\theta_{1}+\theta_{2}\right)}+a_{25} \mathrm{e}^{\mathrm{i}\left(\theta_{1}-\theta_{2}\right)}+\text { c.c. }+\cdots, \\
\phi\left(z, \theta_{1}, \theta_{2}\right)= & b_{1} \mathrm{e}^{v_{1} z+\mathrm{i} \theta_{1}}+b_{2} \mathrm{e}^{v_{2} z+\mathrm{i} \theta_{2}}+b_{22} \mathrm{e}^{2\left(v_{1} z+\mathrm{i} \theta_{1}\right)}+b_{23} \mathrm{e}^{2\left(v_{2} z+\mathrm{i} \theta_{2}\right)} \\
& +b_{24} \mathrm{e}^{\chi+z+\mathrm{i}\left(\theta_{1}+\theta_{2}\right)}+b_{25} \mathrm{e}^{\chi-z+\mathrm{i}\left(\theta_{1}-\theta_{2}\right)}+\text { c.c. }+\cdots,
\end{aligned}
$$


where

$$
\begin{array}{ll}
v_{1}=\left(k_{1}^{2}+\ell_{1}^{2}\right)^{1 / 2}, & \chi_{+}=\left[\left(k_{1}+k_{2}\right)^{2}+\left(\ell_{1}+\ell_{2}\right)^{2}\right]^{1 / 2}, \\
v_{2}=\left(k_{2}^{2}+\ell_{2}^{2}\right)^{1 / 2}, & \chi_{-}=\left[\left(k_{1}-k_{2}\right)^{2}+\left(\ell_{1}-\ell_{2}\right)^{2}\right]^{1 / 2} .
\end{array}
$$

Define $\cos \gamma=\left(k_{1} k_{2}+\ell_{1} \ell_{2}\right) /\left(v_{1} v_{2}\right)$. The angle $\gamma$ is the angle between the wave vectors $\left(k_{1}, \ell_{1}\right)$ and $\left(k_{2}, \ell_{2}\right)$. In terms of $\gamma$,

$$
\chi_{+}^{2}=v_{1}^{2}+v_{2}^{2}+2 \cos \gamma v_{1} v_{2}, \quad \chi_{-}^{2}=v_{1}^{2}+v_{2}^{2}-2 \cos \gamma v_{1} v_{2} .
$$

The above expressions for $\eta, \phi, \Phi, u$ and $v$ are substituted into the definitions of the functionals $\mathscr{S}, \mathscr{A}_{j}, \mathscr{B}_{j}$ and $\mathscr{C}_{j}$ for $j=1,2$ in order to construct the functional $\mathscr{F}\left(a_{1}, b_{1}, \ldots, \omega, k, \ell\right)$. The Fourier coefficients $b_{1}, b_{2}, \ldots$ and $a_{22}, a_{23}, \ldots$ are eliminated using

$$
\frac{\partial \mathscr{F}}{\partial \bar{b}_{1}}=0, \frac{\partial \mathscr{F}}{\partial \bar{b}_{2}}=0, \ldots, \quad \frac{\partial \mathscr{F}}{\partial \bar{a}_{22}}=0, \frac{\partial \mathscr{F}}{\partial \bar{a}_{23}}=0, \ldots,
$$

resulting in $a_{21}=0$ to leading order and

$$
\begin{aligned}
b_{1} & =\mathrm{i} \frac{\omega_{1}}{v_{1}} A_{1}+\cdots, & b_{2} & =\mathrm{i} \frac{\omega_{2}}{v_{2}} A_{2}+\cdots, \\
b_{22} & =0+\cdots, & b_{23} & =0+\cdots, \\
b_{24} & =\mathrm{i} \widehat{b_{24}} A_{1} A_{2}+\cdots, & b_{25} & =\mathrm{i} \widehat{b_{25}} A_{1} \overline{A_{2}}+\cdots, \\
a_{22} & =v_{1} A_{1}^{2}+\cdots, & a_{23} & =v_{2} A_{2}^{2}+\cdots, \\
a_{24} & =\widehat{a_{24}} A_{1} A_{2}+\cdots, & a_{25} & =\widehat{a_{25}} A_{1} \overline{A_{2}}+\cdots,
\end{aligned}
$$

where

$$
\begin{aligned}
& \widehat{a_{24}}=\frac{1}{4} \chi_{+} \mathrm{K}_{+}+\left(v_{1}+v_{2}\right)-\frac{2}{g} \omega_{1} \omega_{2} \sin ^{2} \frac{1}{2} \gamma, \\
& \widehat{a_{25}}=\frac{1}{4} \chi_{-} \mathrm{K}_{-}+\left(v_{1}+v_{2}\right)+\frac{2}{g} \omega_{1} \omega_{2} \cos ^{2} \frac{1}{2} \gamma, \\
& \widehat{b_{24}}=\frac{1}{4}\left(\omega_{1}+\omega_{2}\right) \mathrm{K}_{+}, \\
& \widehat{b_{25}}=\frac{1}{4}\left(\omega_{1}-\omega_{2}\right) \mathrm{K}_{-},
\end{aligned}
$$

and

$$
\mathrm{K}_{+}=\frac{16 \omega_{1} \omega_{2} \sin ^{2} \frac{1}{2} \gamma}{\left[g \chi_{+}-\left(\omega_{1}+\omega_{2}\right)^{2}\right]}, \quad \mathrm{K}_{-}=-\frac{16 \omega_{1} \omega_{2} \cos ^{2} \frac{1}{2} \gamma}{\left[g \chi_{-}-\left(\omega_{1}-\omega_{2}\right)^{2}\right]} .
$$

Back substitution into $\mathscr{F}$ results in the reduced functional

$$
\begin{aligned}
\widehat{\mathscr{F}}\left(\left|A_{1}\right|^{2},\left|A_{2}\right|^{2}, \omega, k, \ell\right)= & \left(\frac{\omega_{1}^{2}}{v_{1}}-g\right)\left|A_{1}\right|^{2}+\left(\frac{\omega_{2}^{2}}{v_{2}}-g\right)\left|A_{2}\right|^{2}+ \\
& -2 v_{1} \omega_{1}^{2}\left|A_{1}\right|^{4}-2 v_{2} \omega_{2}^{2}\left|A_{2}\right|^{4}+\Upsilon\left|A_{1}\right|^{2}\left|A_{2}\right|^{2}+\cdots,
\end{aligned}
$$

where

$$
\begin{aligned}
\Upsilon= & \frac{\omega_{1} \omega_{2}}{g}\left(\omega_{1}+\omega_{2}\right)^{2} \mathrm{~K}_{+} \sin ^{2} \frac{1}{2} \gamma-\frac{\omega_{1} \omega_{2}}{g}\left(\omega_{1}-\omega_{2}\right)^{2} \mathrm{~K}_{-} \cos ^{2} \frac{1}{2} \gamma \\
& -8 \omega_{1} \omega_{2}\left(v_{1}+v_{2}\right) \cos \gamma+\frac{2}{g} \omega_{1}^{2} \omega_{2}^{2}\left(3+\cos ^{2} \gamma\right) .
\end{aligned}
$$


Taking the gradient of $\widehat{\mathscr{F}}$ with respect to $\bar{A}_{1}$ and $\bar{A}_{2}$ results in

$$
\left.\begin{array}{l}
{\left[\left(\omega_{1}^{2} / v_{1}-g\right)-4 v_{1} \omega_{1}^{2}\left|A_{1}\right|^{2}+\Upsilon\left|A_{2}\right|^{2}+\cdots\right] A_{1}=0,} \\
{\left[\left(\omega_{2}^{2} / v_{2}-g\right)+\Upsilon\left|A_{1}\right|^{2}-4 v_{2} \omega_{2}^{2}\left|A_{2}\right|^{2}+\cdots\right] A_{2}=0,}
\end{array}\right\}
$$

which is in the standard form (4.1).

First note that if $\left|A_{2}\right|=0$ and $\left|A_{1}\right| \neq 0$ or $\left|A_{1}\right|=0$ and $\left|A_{2}\right| \neq 0$, we recover the weakly nonlinear dispersion relation for a plane monochromatic wave. When $\left|A_{1}\right| \cdot\left|A_{2}\right| \neq 0$ the nonlinear frequency change as a function of amplitude for the (generically) quasiperiodic two-wave interaction is obtained.

The coefficients in (4.4) agree with existing results on the two-wave interaction (Longuet-Higgins 1962; Weber \& Barrick 1977; Willebrand 1975; Pierson 1993), and when the SCW limit is taken, the coefficient $\Upsilon$ reduces to the coefficient $b$ in (1.6) for SCWs which agrees with the expression for SCWs in Bridges, Dias \& Menasce (2001) (denoted $\alpha_{3}$ on their p. 165). An explicit expression for the SCW limit of $\Upsilon$ is given in $\S 9$.

\section{Linear stability problem for the oblique two-wave interaction}

Take the governing equations in the form (3.1) and suppose there exists a smooth six-parameter family of two-phase waves as in $\S 3$. Consider a perturbation of this basic state of the form $Z \mapsto \widehat{Z}+Z$ and linearize (3.1) about the basic state. The result is the linear system of PDEs

$$
\mathbf{M} \frac{\partial Z}{\partial t}+\mathbf{K} \frac{\partial Z}{\partial x}+\mathbf{L} \frac{\partial Z}{\partial y}=\operatorname{Hess}_{Z} S(\widehat{Z}) Z,
$$

where $\operatorname{Hess}_{Z} S(\widehat{Z})$ is the Hessian of $S(Z)$ evaluated at $\widehat{Z}$.

Consider the following class of perturbations

$$
Z\left(\theta_{1}, \theta_{2}, x, y, t\right)=\operatorname{Re}\left\{U\left(\theta_{1}, \theta_{2}\right) \mathrm{e}^{\mathrm{i}(\alpha x+\beta y+\Omega t)}\right\},
$$

with $\alpha$ and $\beta$ real, $\Omega \in \mathbb{C}$, and $U\left(\theta_{1}, \theta_{2}\right)$ a $2 \pi$-periodic function of $\theta_{1}$ and $\theta_{2}$. Substitution results in the following eigenvalue problem for the stability exponent $\Omega \in \mathbb{C}$,

$$
\mathscr{L} U=\mathrm{i} \Omega \mathbf{M} U+\mathrm{i} \alpha \mathbf{K} U+\mathrm{i} \beta \mathbf{L} U
$$

where

$$
\begin{aligned}
\mathscr{L} & =\operatorname{Hess}_{Z} S(\widehat{Z})-\mathbf{J}_{1} \frac{\partial}{\partial \theta_{1}}-\mathbf{J}_{2} \frac{\partial}{\partial \theta_{2}} \\
& =\operatorname{Hess}_{Z} S(\widehat{Z})-\omega_{1} \mathbf{M} \frac{\partial}{\partial \theta_{1}}-\omega_{2} \mathbf{M} \frac{\partial}{\partial \theta_{2}}-k_{1} \mathbf{K} \frac{\partial}{\partial \theta_{1}}-k_{2} \mathbf{K} \frac{\partial}{\partial \theta_{2}}-\ell_{1} \mathbf{L} \frac{\partial}{\partial \theta_{1}}-\ell_{2} \mathbf{L} \frac{\partial}{\partial \theta_{2}} \\
& =\operatorname{Hess}_{Z} \mathscr{F}(\widehat{Z}),
\end{aligned}
$$

using (3.4) and the definition of $\mathscr{F}$ in (3.7).

Attention will be restricted to long-wave instabilities where $|\alpha|^{2}+|\beta|^{2} \ll 1$. This hypothesis does not put any restriction on the amplitude of the basic state, it restricts only the class of perturbations. When $\alpha=\beta=0$, the eigenvalue problem for $\Omega$ has (at least) a double zero eigenvalue because the kernel of $\mathscr{L}$ is non-trivial. The strategy is to expand the solution of (5.3) in a Taylor series in $\alpha$ and $\beta$. Then a solvability condition will lead to the leading-order behaviour of the stability exponent. 
When $\Omega=\alpha=\beta=0,(5.3)$ has two solutions,

$$
\operatorname{Ker}(\mathscr{L})=\operatorname{span}\left\{\boldsymbol{\psi}_{1}, \boldsymbol{\psi}_{2}\right\} \quad \text { where } \quad \boldsymbol{\psi}_{j}=\frac{\partial \widehat{Z}}{\partial \theta_{j}} \quad \text { for } j=1,2 .
$$

This follows since differentiation of (3.4) with respect to $\theta_{1}$ and $\theta_{2}$ results in $\mathscr{L}\left(\partial_{\theta_{j}} \widehat{Z}\right)=0, j=1,2$. Therefore $\operatorname{Ker}(\mathscr{L}) \supseteq \operatorname{span}\left\{\boldsymbol{\psi}_{1}, \boldsymbol{\psi}_{2}\right\}$. For particular parameter values (or with additional symmetry), the kernel may be larger, but generically we have equality, and this is assumed hereinafter.

The general solution of (5.3) can be expressed in the following form to leading order

$$
\begin{aligned}
U= & c_{1} U_{1}+c_{2} U_{2} \\
= & c_{1}\left(\psi_{1}+\mathrm{i} \alpha \frac{\partial \widehat{Z}}{\partial k_{1}}+\mathrm{i} \beta \frac{\partial \widehat{Z}}{\partial \ell_{1}}+\mathrm{i} \Omega \frac{\partial \widehat{Z}}{\partial \omega_{1}}\right) \\
& +c_{2}\left(\psi_{2}+\mathrm{i} \alpha \frac{\partial \widehat{Z}}{\partial k_{2}}+\mathrm{i} \beta \frac{\partial \widehat{Z}}{\partial \ell_{2}}+\mathrm{i} \Omega \frac{\partial \widehat{Z}}{\partial \omega_{2}}\right)+O\left(|\Omega|^{2}+|\alpha|^{2}+|\beta|^{2}\right),
\end{aligned}
$$

where $\left(c_{1}, c_{2}\right)$ are at present arbitrary complex constants whose properties are to be determined as part of the analysis. This form of the leading-order solution is confirmed by noting that differentiation of (1.1) results in

$$
\mathscr{L}\left(\frac{\partial \widehat{Z}}{\partial \omega_{j}}\right)=\mathbf{M} \boldsymbol{\psi}_{j}, \quad \mathscr{L}\left(\frac{\partial \widehat{Z}}{\partial k_{j}}\right)=\mathbf{K} \boldsymbol{\psi}_{j}, \quad \mathscr{L}\left(\frac{\partial \widehat{Z}}{\partial \ell_{j}}\right)=\mathbf{L} \boldsymbol{\psi}_{j} \quad \text { for } j=1,2 .
$$

An expression for the stability exponent is obtained by using (5.5) and applying the solvability condition to (5.3). Introduce the following inner product for functions $Z \in \mathbb{H}$ that are $2 \pi$-periodic in $\theta_{1}$ and $\theta_{2}$,

$\llbracket U, V \rrbracket=\oint\left\langle U\left(\theta_{1}, \theta_{2}\right), V\left(\theta_{1}, \theta_{2}\right)\right\rangle \mathrm{d} \theta=\frac{1}{(2 \pi)^{2}} \int_{0}^{2 \pi} \int_{0}^{2 \pi}\left\langle U\left(\theta_{1}, \theta_{2}\right), V\left(\theta_{1}, \theta_{2}\right)\right\rangle \mathrm{d} \theta_{1} \mathrm{~d} \theta_{2}$,

where $\langle\cdot, \cdot\rangle$ is the inner product on $\mathbb{H}$. Since $\operatorname{Ker}(\mathscr{L})=\operatorname{span}\left\{\boldsymbol{\psi}_{1}, \boldsymbol{\psi}_{2}\right\}$ by hypothesis and $\mathscr{L}$ is formally symmetric, we have the following two solvability conditions for (5.3),

$$
\llbracket \psi_{j},(\mathrm{i} \alpha \mathbf{K}+\mathrm{i} \beta \mathbf{L}+\mathrm{i} \Omega \mathbf{M}) U \rrbracket=0 \quad \text { for } \quad j=1,2 \text {. }
$$

However, since (5.3) is linear and $U=c_{1} U_{1}+c_{2} U_{2}$, the solvability condition is equivalent to

$$
\mathbf{N}(\Omega, \alpha, \beta) c=\mathbf{0}
$$

where $\mathbf{N}(\Omega, \alpha, \beta)$ is the $2 \times 2$ matrix

$$
\begin{aligned}
& \mathbf{N}(\Omega, \alpha, \beta) \\
& \quad=\left[\begin{array}{ll}
\llbracket \boldsymbol{\psi}_{1},(\mathrm{i} \alpha \mathbf{K}+\mathrm{i} \beta \mathbf{L}+\mathrm{i} \Omega \mathbf{M}) U_{1} \rrbracket & \llbracket \boldsymbol{\psi}_{1},(\mathrm{i} \alpha \mathbf{K}+\mathrm{i} \beta \mathbf{L}+\mathrm{i} \Omega \mathbf{M}) U_{2} \rrbracket \\
\llbracket \boldsymbol{\psi}_{2},(\mathrm{i} \alpha \mathbf{K}+\mathrm{i} \beta \mathbf{L}+\mathrm{i} \Omega \mathbf{M}) U_{1} \rrbracket & \llbracket \boldsymbol{\psi}_{2},(\mathrm{i} \alpha \mathbf{K}+\mathrm{i} \beta \mathbf{L}+\mathrm{i} \Omega \mathbf{M}) U_{2} \rrbracket
\end{array}\right] \text { and } \boldsymbol{c}=\left(\begin{array}{l}
c_{1} \\
c_{2}
\end{array}\right) .
\end{aligned}
$$

The matrix $\mathbf{N}(\Omega, \alpha, \beta)$ has complex-valued entries dependent on $\Omega \in \mathbb{C}$ and $(\alpha, \beta) \in$ $\mathbb{R}^{2}$. Clearly, there is a non-trivial solution, i.e. $\|\boldsymbol{c}\| \neq 0$, of the linear stability problem if and only if

$$
\Delta(\Omega, \alpha, \beta) \stackrel{\operatorname{def}}{=} \operatorname{det}[\mathbf{N}(\Omega, \alpha, \beta)]=0 .
$$


This leads to the following definition of instability: If for some $(\alpha, \beta) \in \mathbb{R}^{2}$ there exists an $\Omega \in \mathbb{C}$ such that $\Delta(\Omega, \alpha, \beta)=0$ and $\operatorname{Im}(\Omega) \neq 0$ the basic state is linearly unstable.

An unstable eigenfunction is constructed as follows. For some $(\alpha, \beta)$, suppose $\Delta(\Omega, \alpha, \beta)$ has an unstable root $\Omega$. Then substitute this $(\alpha, \beta, \Omega)$ into the expression for $U\left(\theta_{1}, \theta_{2}\right)$ which in turn is substituted into the expression for the perturbation (5.2). The resulting function $Z\left(\theta_{1}, \theta_{2}, x, y, t\right)$ is then an approximation to an unstable eigenfunction of (5.1), valid for $|\Omega|,|\alpha|$ and $|\beta|$ sufficiently small.

The expression for $U$ in (5.5) is used to construct the leading-order Taylor expansion of $\mathbf{N}(\Omega, \alpha, \beta)$, and hence $\Delta(\Omega, \alpha, \beta)$, to obtain a sufficient condition for linear instability valid for $|\Omega|^{2}+|\alpha|^{2}+|\beta|^{2}$ sufficiently small.

Our main result is that the matrix $\mathbf{N}$ can be expressed in terms of known quantities. First, the expression will be given, and then it will be verified,

$$
\mathbf{N}(\Omega, \alpha, \beta)=\mathbf{N}_{0}+\mathbf{N}_{1} \Omega+\mathbf{N}_{2} \Omega^{2}+o\left(|\Omega|^{2}+|\alpha|^{2}+|\beta|^{2}\right),
$$

with

$$
\left.\begin{array}{l}
\mathbf{N}_{0}=\alpha^{2} \frac{\delta \mathscr{B}}{\delta k}+\alpha \beta\left(\frac{\delta \mathscr{B}}{\delta \ell}+\frac{\delta \mathscr{C}}{\delta k}\right)+\beta^{2} \frac{\delta \mathscr{C}}{\delta \ell}, \\
\mathbf{N}_{1}=\alpha\left(\frac{\delta \mathscr{A}}{\delta k}+\frac{\delta \mathscr{B}}{\delta \omega}\right)+\beta\left(\frac{\delta \mathscr{A}}{\delta \ell}+\frac{\delta \mathscr{C}}{\delta \omega}\right), \\
\mathbf{N}_{2}=\frac{\delta \mathscr{A}}{\delta \omega},
\end{array}\right\}
$$

where the matrices $\delta \mathscr{A} / \delta \omega$ etc. are defined in (3.11). The Jacobians from the variational principle of $\S 3$ appear in a central way in the analysis of long-wave instability: the leading-order terms in the stability problem can be obtained from known information about the basic state.

The derivation of the entries of the matrix $\mathbf{N}_{2}$ is given, with the verification of the other two following the same argument. By definition

$$
\mathbf{N}(\Omega, 0,0)=\left(\begin{array}{ll}
\mathrm{i} \Omega \llbracket \boldsymbol{\psi}_{1}, \mathbf{M} U_{1} \rrbracket & \mathrm{i} \Omega \llbracket \boldsymbol{\psi}_{1}, \mathbf{M} U_{2} \rrbracket \\
\mathrm{i} \Omega \llbracket \boldsymbol{\psi}_{2}, \mathbf{M} U_{1} \rrbracket & \mathrm{i} \Omega \llbracket \boldsymbol{\psi}_{2}, \mathbf{M} U_{2} \rrbracket
\end{array}\right) .
$$

Substitute the leading-order expression for $U_{1}$ and $U_{2}$ from (5.5),

$$
\begin{aligned}
\mathbf{N}(\Omega, 0,0) & =\left(\begin{array}{ll}
\mathrm{i} \Omega \llbracket \boldsymbol{\psi}_{1}, \mathbf{M}\left(\boldsymbol{\psi}_{1}+\mathrm{i} \Omega \widehat{Z}_{\omega_{1}}\right) \rrbracket & \mathrm{i} \Omega \llbracket \boldsymbol{\psi}_{1}, \mathbf{M}\left(\boldsymbol{\psi}_{2}+\mathrm{i} \Omega \widehat{Z}_{\omega_{2}}\right) \rrbracket \\
\mathrm{i} \Omega \llbracket \boldsymbol{\psi}_{2}, \mathbf{M}\left(\boldsymbol{\psi}_{1}+\mathrm{i} \Omega \widehat{Z}_{\omega_{1}}\right) \rrbracket & \mathrm{i} \Omega \llbracket \boldsymbol{\psi}_{2}, \mathbf{M}\left(\boldsymbol{\psi}_{2}+\mathrm{i} \Omega \widehat{Z}_{\omega_{2}}\right) \rrbracket
\end{array}\right) \\
& =\Omega^{2}\left(\begin{array}{ll}
\llbracket \mathbf{M} \boldsymbol{\psi}_{1}, \widehat{Z}_{\omega_{1}} \rrbracket & \llbracket \mathbf{M} \boldsymbol{\psi}_{1}, \widehat{Z}_{\omega_{2}} \rrbracket \\
\llbracket \mathbf{M} \boldsymbol{\psi}_{2}, \widehat{Z}_{\omega_{1}} \rrbracket & \llbracket \mathbf{M} \boldsymbol{\psi}_{2}, \widehat{Z}_{\omega_{2}} \rrbracket
\end{array}\right),
\end{aligned}
$$

where we have used the identities $\llbracket \boldsymbol{\psi}_{i}, \mathbf{M} \boldsymbol{\psi}_{j} \rrbracket=0$ for $i, j=1,2$. Now, let $\mathscr{A}_{j}(\widehat{Z})$ be the actions (3.5) evaluated at the basic state. Then

$$
\frac{\delta \mathscr{A}}{\delta \omega}=\left[\begin{array}{cc}
\frac{\partial \mathscr{A}_{1}}{\partial \omega_{1}} & \frac{\partial \mathscr{A}_{1}}{\partial \omega_{2}} \\
\frac{\partial \mathscr{A}_{2}}{\partial \omega_{1}} & \frac{\partial \mathscr{A}_{2}}{\partial \omega_{2}}
\end{array}\right]=\left(\begin{array}{ll}
\llbracket \mathbf{M} \partial_{\theta_{1}} \widehat{Z}, \partial_{\omega_{1}} \widehat{Z} \rrbracket & \llbracket \mathbf{M} \partial_{\theta_{1}} \widehat{Z}, \partial_{\omega_{2}} \widehat{Z} \rrbracket \\
\llbracket \mathbf{M} \partial_{\theta_{2}} \widehat{Z}, \partial_{\omega_{1}} \widehat{Z} \rrbracket & \llbracket \mathbf{M} \partial_{\theta_{2}} \widehat{Z}, \partial_{\omega_{2}} \widehat{Z} \rrbracket
\end{array}\right) .
$$


Comparing the above two results proves that

$$
\mathbf{N}(\Omega, 0,0)=\Omega^{2} \frac{\delta \mathscr{A}}{\delta \omega} .
$$

The leading-order part of $\mathbf{N}$ in (5.9) is of the form of a lambda matrix (Lancaster 1966). Solving for the $\Omega$ roots, is equivalent to solving the nonlinear in the parameter eigenvalue problem,

$$
\left[\mathbf{N}_{0}+\mathbf{N}_{1} \Omega+\mathbf{N}_{2} \Omega^{2}\right] c=\mathbf{0},
$$

for the eigenvalue $\Omega$ and eigenvector $\boldsymbol{c}$. These quadratic eigenvalue problems frequently arise in the theory of vibrating systems in mechanics. This quadratic eigenvalue problem is equivalent (when $\operatorname{det}\left(\mathbf{N}_{2}\right) \neq 0$ ) to the problem of finding the eigenvalues $\Omega$ of the classical generalized symmetric eigenvalue problem

$$
\left[\left(\begin{array}{cc}
\mathbf{0} & \mathbf{N}_{2} \\
\mathbf{N}_{2} & \mathbf{N}_{1}
\end{array}\right) \Omega+\left(\begin{array}{cc}
-\mathbf{N}_{2} & \mathbf{0} \\
\mathbf{0} & \mathbf{N}_{0}
\end{array}\right)\right]\left(\begin{array}{l}
\boldsymbol{d} \\
\boldsymbol{c}
\end{array}\right)=\left(\begin{array}{l}
\mathbf{0} \\
\mathbf{0}
\end{array}\right)
$$

(Lancaster 1966, pp. 58-59). However, we have found no advantage to studying this linear eigenvalue problem, rather than the nonlinear form.

The main result of this section is: given a basic state $\left(\widehat{Z}, \omega_{1}, k_{1}, \ell_{1}, \omega_{2}, k_{2}, \ell_{2}\right)$, there are accompanying Jacobian matrices $\delta \mathscr{A} / \delta \omega, \ldots, \delta \mathscr{C} / \delta \ell$ which arise naturally in the variational principle of $\S 3$, and the long-wave instability is completely determined by these Jacobian matrices. This stability result is for the general oblique two-wave interaction. A special case is a stability result for SCWs.

\section{The stability quartic for long-wave instabilities}

Expanding the determinant $\Delta(\Omega, \alpha, \beta)$ leads to a quartic polynomial for the stability exponent $\Omega$

$$
\Delta(\Omega, \alpha, \beta)=\operatorname{det}\left[\mathbf{N}_{0}+\mathbf{N}_{1} \Omega+\mathbf{N}_{2} \Omega^{2}\right]=g_{4} \Omega^{4}+g_{3} \Omega^{3}+g_{2} \Omega^{2}+g_{1} \Omega+g_{0},
$$

where

$$
\begin{aligned}
& g_{4}= \operatorname{det}\left(\frac{\delta \mathscr{A}}{\delta \omega}\right), \\
& g_{3}=\operatorname{tr}\left[\left(\frac{\delta \mathscr{A}}{\delta \omega}\right)^{\sharp}\left(\alpha\left[\frac{\delta \mathscr{A}}{\delta k}+\frac{\delta \mathscr{B}}{\delta \omega}\right]+\beta\left[\frac{\delta \mathscr{A}}{\delta \ell}+\frac{\delta \mathscr{C}}{\delta \omega}\right]\right)\right], \\
& g_{2}=\operatorname{det}\left(\alpha\left[\frac{\delta \mathscr{A}}{\delta k}+\frac{\delta \mathscr{B}}{\delta \omega}\right]+\beta\left[\frac{\delta \mathscr{A}}{\delta \ell}+\frac{\delta \mathscr{C}}{\delta \omega}\right]\right) \\
&+\operatorname{tr}\left[\left(\frac{\delta \mathscr{A}}{\delta \omega}\right)^{\sharp}\left(\alpha^{2} \frac{\delta \mathscr{B}}{\delta k}+\alpha \beta\left[\frac{\delta \mathscr{B}}{\delta \ell}+\frac{\delta \mathscr{C}}{\delta k}\right]+\beta^{2} \frac{\delta \mathscr{C}}{\delta \ell}\right)\right], \\
& g_{1}=\operatorname{tr}\left[\left(\alpha^{2} \frac{\delta \mathscr{B}}{\delta k}+\alpha \beta\left[\frac{\delta \mathscr{B}}{\delta \ell}+\frac{\delta \mathscr{C}}{\delta k}\right]+\beta^{2} \frac{\delta \mathscr{C}}{\delta \ell}\right)^{\sharp}\left(\alpha\left[\frac{\delta \mathscr{A}}{\delta k}+\frac{\delta \mathscr{B}}{\delta \omega}\right]+\beta\left[\frac{\delta \mathscr{A}}{\delta \ell}+\frac{\delta \mathscr{C}}{\delta \omega}\right]\right)\right], \\
& g_{0}=\operatorname{det}\left(\alpha^{2} \frac{\delta \mathscr{B}}{\delta k}+\alpha \beta\left[\frac{\delta \mathscr{B}}{\delta \ell}+\frac{\delta \mathscr{C}}{\delta k}\right]+\beta^{2} \frac{\delta \mathscr{C}}{\delta \ell}\right) .
\end{aligned}
$$

where $\operatorname{tr}()$ is the trace, and the superscript $\sharp$ indicates adjugate. The it adjugate of a matrix is the transpose of the cofactor matrix. If a matrix $\mathbf{R}$ is invertible then $\mathbf{R}^{\sharp}=\operatorname{det}(\mathbf{R}) \mathbf{R}^{-1}$. 


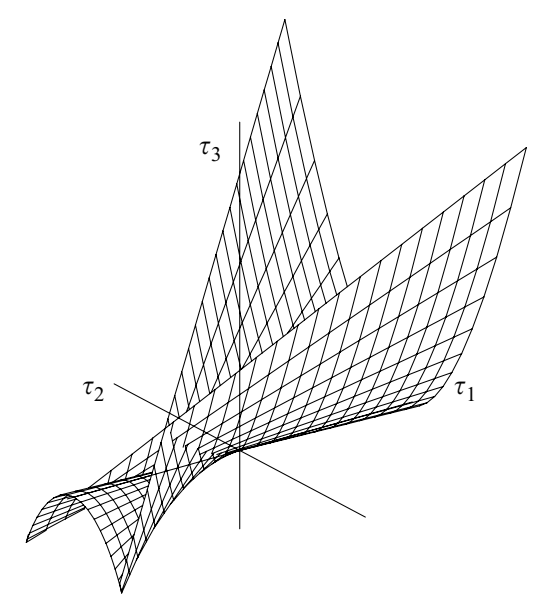

FIGURE 4. The discriminant surface for the quartic.

Dividing through the quartic by $g_{4}$ and introducing the transformation $\Omega=X-$ $\left(g_{3} / 4\right) / g_{4}$ reduces the quartic to standard form,

$$
\Delta(X)=X^{4}+\tau_{1} X^{2}+\tau_{2} X+\tau_{3},
$$

with

$$
\left.\begin{array}{rl}
\tau_{1} & =\frac{1}{g_{4}^{2}}\left(-\frac{3}{8} g_{3}^{2}+g_{4} g_{2}\right), \\
\tau_{2} & =\frac{1}{g_{4}^{3}}\left(\frac{1}{8} g_{3}^{3}-\frac{1}{2} g_{4} g_{3} g_{2}+g_{4}^{2} g_{1}\right), \\
\tau_{3} & =\frac{1}{g_{4}^{4}}\left(-\frac{3}{256} g_{3}^{4}+\frac{1}{16} g_{4} g_{3}^{2} g_{2}-\frac{1}{4} g_{4}^{2} g_{3} g_{1}+g_{4}^{3} g_{0}\right),
\end{array}\right\}
$$

Since $g_{3}$ and $g_{4}$ are real, the $\operatorname{Im}(\Omega) \neq 0$ if and only if $\operatorname{Im}(X) \neq 0$. Therefore, we can appeal to standard results for the quartic to determine when there is at least one zero of $\Delta(X)$ with non-zero imaginary part.

There are three diagnostic functions

$$
d_{1}=\tau_{1}, \quad d_{2}=\text { Discriminant }, \quad d_{3}=\tau_{1}^{2}-4 \tau_{3} .
$$

where Discriminant $=16 \tau_{3} \tau_{1}^{4}-4 \tau_{2}^{2} \tau_{1}^{3}-128 \tau_{3}^{2} \tau_{1}^{2}+144 \tau_{2}^{2} \tau_{3} \tau_{1}-27 \tau_{2}^{4}+256 \tau_{3}^{3}$. The conditions for instability (the existence of at least one root with non-zero imaginary part) are

$$
d_{1}>0 \quad \text { or } d_{1}<0, \quad d_{2}<0 \quad \text { or } \quad d_{1}<0, \quad d_{2}>0, \quad d_{3}<0 .
$$

The discriminant surface is plotted in $\tau$ space in figure 4. If $\tau_{1}>0$ it is immediate that there is at least one unstable eigenvalue. When $\tau_{1}<0$ we must check additional diagnostics. A section through the discriminant surface for $\tau_{1}<0$ is shown in figure 5 . Unless $\tau_{2}$ and $\tau_{3}$ are in the enclosed central region (marked with a 4 in the figure) there is a root which is unstable (having a non-zero imaginary part).

Therefore, given a basic state with associated Jacobian matrices, the problem of long-wave instability reduces to checking the above conditions on the quartic. The problem of long-wave instability for SCWs reduces to checking the above conditions after the SCW limit is taken. This programme will be carried out for weakly nonlinear SCWs in the next section. 


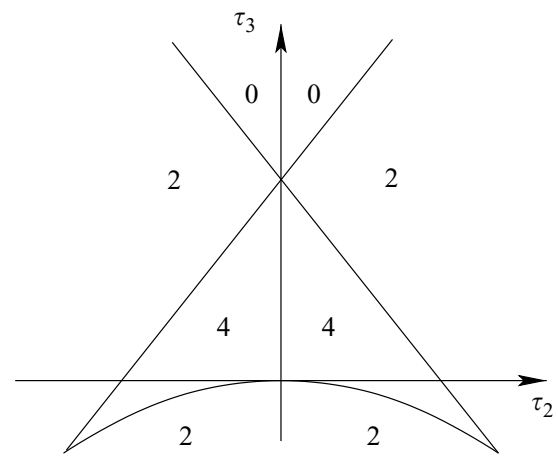

FIGURE 5. A constant $\tau_{1}$ section through the discriminant surface with $\tau_{1}<0$. The number of real roots of the quartic in each region of parameter space is labelled 0,2 or 4.

\section{Long-wave instability of weakly nonlinear SCWs}

A general theory for long-wave instability of weakly nonlinear SCWs is now given, starting with the results for the weakly nonlinear two-wave interaction.

$$
Z(x, y, t)=A_{1} \xi_{1} \mathrm{e}^{\mathrm{i}\left(k_{1} x+\ell_{1} y+\omega_{1} t\right)}+A_{2} \xi_{2} \mathrm{e}^{\mathrm{i}\left(k_{2} x+\ell_{2} y+\omega_{2} t\right)}+\text { c.c. }+\cdots,
$$

where $\boldsymbol{\xi}_{j}$ satisfies

$$
\left[\operatorname{Hess}_{Z} S(0)-\mathrm{i} k_{j} \mathbf{K}-\mathrm{i} \ell_{j} \mathbf{L}-\mathrm{i} \omega_{j} \mathbf{M}\right] \boldsymbol{\xi}_{j}=0,
$$

with associated dispersion relation $D\left(\omega_{j}, k_{j}, \ell_{j}\right)=0$. When the phase space is finite dimensional (such as the nonlinear wave equation in $\$ 2.1$ ), the dispersion relation is defined by

$$
D(\omega, k, \ell)=\operatorname{det}\left[\operatorname{Hess}_{Z} S(0)-\mathrm{i} k \mathbf{K}-\mathrm{i} \ell \mathbf{L}-\mathrm{i} \omega \mathbf{M}\right],
$$

and in infinite dimensions it is the condition for solvability of (7.1).

The reduced equation which generalizes (4.2) is

$$
\begin{aligned}
\widehat{\mathscr{F}}\left(\left|A_{1}\right|^{2},\left|A_{2}\right|^{2}, \omega, k, \ell\right)= & D\left(\omega_{1}, k_{1}, \ell_{1}\right)\left|A_{1}\right|^{2}+D\left(\omega_{2}, k_{2}, \ell_{2}\right)\left|A_{2}\right|^{2} \\
& +\frac{1}{2} \Lambda_{11}\left|A_{1}\right|^{4}+\Lambda_{12}\left|A_{1}\right|^{2}\left|A_{2}\right|^{2}+\frac{1}{2} \Lambda_{22}\left|A_{2}\right|^{4}+\cdots .
\end{aligned}
$$

By construction, the two-wave interaction determined by this reduced equation is a deformation of a family of SCWs. Therefore, in the SCW limit,

$$
k_{2}=k_{1}=k, \quad \ell_{2}=-\ell_{1}=-\ell, \quad \omega_{2}=\omega_{1}=-\omega, \quad\left|A_{2}\right|=\left|A_{1}\right|=|A|,
$$

the coefficients of the nonlinear quartic terms reduce to

$$
\Lambda_{11} \rightarrow a, \quad \Lambda_{22} \rightarrow a, \quad \Lambda_{12} \rightarrow b,
$$

where $a$ and $b$ are the coefficients associated with SCWs as in (1.6).

In the SCW limit, the quadratic coefficients in (7.2) also simplify. The SCW symmetry (3.2) is inherited by the dispersion relation: $D(\omega, k, \ell)=D(\omega, k,-\ell)$, and the Hamiltonian structure induces the symmetry $D(-\omega,-k,-\ell)=D(\omega, k, \ell)$. Hence the dispersion relation for SCWs can always be expressed in the form

$$
D(\omega, k, \ell)=\widehat{d}\left(\omega^{2}, \omega k, k^{2}, \ell^{2}\right) .
$$

These symmetry properties are useful for evaluating the stability matrices for the general weakly nonlinear SCW stability analysis, without explicitly knowing the dispersion relation.

\section{CAMBridge JDURNALS}


Differentiating $\widehat{\mathscr{F}}$ in (7.2) with respect to the amplitudes, results in the following expression for the amplitudes to leading order

$$
\left(\begin{array}{l}
\left|A_{1}\right|^{2} \\
\left|A_{2}\right|^{2}
\end{array}\right)=-\boldsymbol{\Lambda}^{-1}\left(\begin{array}{c}
D\left(\omega_{1}, k_{1}, \ell_{1}\right) \\
D\left(\omega_{2}, k_{2}, \ell_{2}\right)
\end{array}\right) \quad \text { where } \quad \boldsymbol{\Lambda}=\left[\begin{array}{ll}
\Lambda_{11} & \Lambda_{12} \\
\Lambda_{12} & \Lambda_{22}
\end{array}\right] .
$$

To compute the stability matrices $\delta \mathscr{A} / \delta \omega, \ldots, \delta \mathscr{C} / \delta \ell$, we take as a starting point the abstract form (3.5). To leading order, the general form for $\mathscr{A}_{j}, \mathscr{B}_{j}$ and $\mathscr{C}_{j}, j=1,2$ is

$$
\mathscr{A}_{j}=\frac{\partial D}{\partial \omega_{j}}\left|A_{j}\right|^{2}+\cdots, \quad \mathscr{B}_{j}=\frac{\partial D}{\partial k_{j}}\left|A_{j}\right|^{2}+\cdots, \quad \mathscr{C}_{j}=\frac{\partial D}{\partial \ell_{j}}\left|A_{j}\right|^{2}+\cdots,
$$

where $D$ is a function of $\left(\omega_{j}, k_{j}, \ell_{j}\right)$, and the amplitudes $\left|A_{j}\right|$ are considered functions of $(\omega, k, \ell)$ through $(7.5)$.

The details of the construction and limit process for $\delta \mathscr{A} / \delta \omega$ are given, and then the results for the other stability matrices is summarized,

$$
\begin{aligned}
\frac{\delta \mathscr{A}}{\delta \omega}= & {\left[\begin{array}{ll}
\frac{\partial \mathscr{A}_{1}}{\partial \omega_{1}} & \frac{\partial \mathscr{A}_{1}}{\partial \omega_{2}} \\
\frac{\partial \mathscr{A}_{2}}{\partial \omega_{1}} & \frac{\partial \mathscr{A}_{2}}{\partial \omega_{2}}
\end{array}\right] } \\
= & -\left(\begin{array}{cc}
\partial_{\omega_{1}} D\left(\omega_{1}, k_{1}, \ell_{1}\right) & 0 \\
0 & \partial_{\omega_{2}} D\left(\omega_{2}, k_{2}, \ell_{2}\right)
\end{array}\right) \boldsymbol{\Lambda}^{-1}\left(\begin{array}{cc}
\partial_{\omega_{1}} D\left(\omega_{1}, k_{1}, \ell_{1}\right) & 0 \\
0 & \partial_{\omega_{2}} D\left(\omega_{2}, k_{2}, \ell_{2}\right)
\end{array}\right) \\
& +\left(\begin{array}{cc}
\partial_{\omega_{1} \omega_{1}} D\left(\omega_{1}, k_{1}, \ell_{1}\right)\left|A_{1}\right|^{2} & \\
0 & \partial_{\omega_{2} \omega_{2}} D\left(\omega_{2}, k_{2}, \ell_{2}\right)\left|A_{2}\right|^{2}
\end{array}\right)+\cdots
\end{aligned}
$$

Hereinafter all expressions are evaluated at the SCW limit in (7.3)-(7.4). Evaluating $\delta \mathscr{A} / \delta \omega$ in this limit,

$$
\frac{\delta \mathscr{A}}{\delta \omega}=-\left(D_{\omega}\right)^{2}(\boldsymbol{\Lambda})^{-1}+D_{\omega \omega}|A|^{2}\left(\begin{array}{ll}
1 & 0 \\
0 & 1
\end{array}\right)+\cdots .
$$

The other stability matrices in the SCW limit are

$$
\begin{aligned}
& \frac{\delta \mathscr{A}}{\delta k}=-D_{\omega} D_{k} \boldsymbol{\Lambda}^{-1}+D_{\omega k}|A|^{2}\left(\begin{array}{ll}
1 & 0 \\
0 & 1
\end{array}\right)+\cdots, \\
& \frac{\delta \mathscr{A}}{\delta \ell}=-D_{\omega} D_{\ell} \boldsymbol{\Lambda}^{-1}\left(\begin{array}{cc}
1 & 0 \\
0 & -1
\end{array}\right)+D_{\omega \ell}|A|^{2}\left(\begin{array}{cc}
1 & 0 \\
0 & -1
\end{array}\right)+\cdots, \\
& \frac{\delta \mathscr{B}}{\delta k}=-D_{k}^{2} \boldsymbol{\Lambda}^{-1}+D_{k k}|A|^{2}\left(\begin{array}{cc}
1 & 0 \\
0 & 1
\end{array}\right)+\cdots, \\
& \frac{\delta \mathscr{B}}{\delta \ell}=-D_{k} D_{\ell} \boldsymbol{\Lambda}^{-1}\left(\begin{array}{cc}
1 & 0 \\
0 & -1
\end{array}\right)+D_{k \ell}|A|^{2}\left(\begin{array}{cc}
1 & 0 \\
0 & -1
\end{array}\right)+\cdots, \\
& \frac{\delta \mathscr{C}}{\delta \ell}=-D_{\ell}^{2} \frac{1}{|\boldsymbol{\Lambda}|} \boldsymbol{\Lambda}+D_{\ell \ell}|A|^{2}\left(\begin{array}{ll}
1 & 0 \\
0 & 1
\end{array}\right)+\cdots,
\end{aligned}
$$

with the other matrices given by the symmetry relations (3.12). 
We are now in a position to compute the coefficients of the stability quartic (6.1)-(6.2),

with

$$
\Delta(\Omega, \alpha, \beta)=g_{4} \Omega^{4}+g_{3} \Omega_{3}+g_{2} \Omega^{2}+g_{1} \Omega+g_{0},
$$

$$
\begin{aligned}
g_{4}= & \frac{D_{\omega}^{4}}{|\boldsymbol{\Lambda}|}-2 a \frac{D_{\omega \omega} D_{\omega}^{2}}{|\boldsymbol{\Lambda}|}|A|^{2}+D_{\omega \omega}^{2}|A|^{4}+\cdots, \\
g_{3}= & 4 \alpha\left[\frac{D_{\omega}^{3} D_{k}}{|\boldsymbol{\Lambda}|}-a \frac{D_{\omega}}{|\boldsymbol{\Lambda}|}\left(D_{\omega} D_{\omega k}+D_{\omega \omega} D_{k}\right)|A|^{2}+D_{\omega \omega} D_{\omega k}|A|^{4}\right]+\cdots, \\
g_{2}= & 2 \frac{D_{\omega}^{2}}{|\boldsymbol{\Lambda}|}\left(3 \alpha^{2} D_{k}^{2}-\beta^{2} D_{\ell}^{2}\right) \\
& -\frac{2 a}{|\boldsymbol{\Lambda}|}\left[\alpha^{2}\left(4 D_{\omega} D_{k} D_{\omega k}+D_{\omega}^{2} D_{k k}+D_{\omega \omega} D_{k}^{2}\right)\right. \\
& \left.+\beta^{2}\left(-4 D_{\omega} D_{\ell} D_{\omega \ell}+D_{\omega \omega} D_{\ell}^{2}+D_{\omega}^{2} D_{\ell \ell}\right)\right]|A|^{2} \\
& +2\left[\alpha^{2}\left(2 D_{\omega k}^{2}+D_{\omega \omega} D_{k k}\right)+\beta^{2}\left(D_{\omega \omega} D_{\ell \ell}-2 D_{\omega \ell}^{2}\right)\right]|A|^{4}+\cdots, \\
g_{1}= & \frac{4}{|\boldsymbol{\Lambda}|}\left[-\alpha D_{\omega} D_{k}\left(\beta D_{\ell}-\alpha D_{k}\right)\left(\beta D_{\ell}+\alpha D_{k}\right)\right] \\
& -\frac{4 a}{|\boldsymbol{\Lambda}|}\left[\alpha^{3}\left(D_{k} D_{\omega k}+D_{\omega} D_{k k}\right) D_{k}\right. \\
& \left.+\alpha \beta^{2}\left(D_{\ell \ell} D_{\omega} D_{k}+D_{\ell}^{2} D_{\omega k}-2 D_{\ell}\left[D_{k \ell} D_{\omega}+D_{k} D_{\omega \ell}\right]\right)\right]|A|^{2} \\
& +4 \alpha\left[\alpha^{2} D_{k k} D_{\omega k}+2 \beta^{2}\left(D_{\ell \ell} D_{\omega k}-D_{k \ell} D_{\omega \ell}\right)\right]|A|^{4}+\cdots, \\
g_{0}= & \frac{1}{|\boldsymbol{\Lambda}|}\left(\beta D_{\ell}-\alpha D_{k}\right)^{2}\left(\beta D_{\ell}+\alpha D_{k}\right)^{2}+ \\
& -2 \frac{a}{|\boldsymbol{\Lambda}|}\left[\alpha^{4} D_{k k} D_{k}^{2}+\alpha^{2} \beta^{2}\left(D_{\ell}^{2} D_{k k}+D_{k}^{2} D_{\ell \ell}-4 D_{\ell} D_{k} D_{k \ell}\right)+\beta^{4} D_{\ell}^{2} D_{\ell \ell}\right]|A|^{2} \\
& +\left(\alpha^{2} D_{k k}-2 \alpha \beta D_{k \ell}+\beta^{2} D_{\ell \ell}\right)\left(\alpha^{2} D_{k k}+2 \alpha \beta D_{k \ell}+\beta^{2} D_{\ell \ell}\right)|A|^{4}+\cdots
\end{aligned}
$$

Details of the calculation of these coefficients can be found in Laine-Pearson (2002).

The only place that the coefficient $b$ in the frequency correction to SCWs appears in the coefficients is in $|\boldsymbol{\Lambda}|=a^{2}-b^{2}$. Multiplying $\Delta(\Omega, \alpha, \beta)$ by $|\boldsymbol{\Lambda}|$ shows that the effect of $b$ does not appear in the coefficients at order $|A|^{0}$ or $|A|^{2}$, but appears in the terms of order $|A|^{4}$.

In the zero-amplitude limit, the stability quartic (7.10) has a nice factorization

$$
\Delta(\Omega, \alpha, \beta)=\frac{1}{|\boldsymbol{\Lambda}|}\left(D_{\omega} \Omega+D_{k} \alpha+D_{\ell} \beta\right)^{2}\left(D_{\omega} \Omega+D_{k} \alpha-D_{\ell} \beta\right)^{2} .
$$

It is clear from this expression that the hypotheses

$$
D_{\omega} \neq 0, \quad D_{k} \neq 0, \quad D_{\ell} \neq 0,
$$

when evaluated at the SCW frequency and wavenumbers, are required to avoid degeneracy. When the conditions (7.12) are not satisfied, it is an indication that resonances or other degeneracies of the dispersion relation will occur.

The eight eigenvalues $\lambda= \pm \mathrm{i} \Omega$ with $\Omega$ a root of (7.11) are purely imaginary and are grouped into four pairs. Their location on the imaginary axis depends on the values of $k$ and $\ell$. In figure 6 , the principal cases are shown. When $\beta=0$, the four 

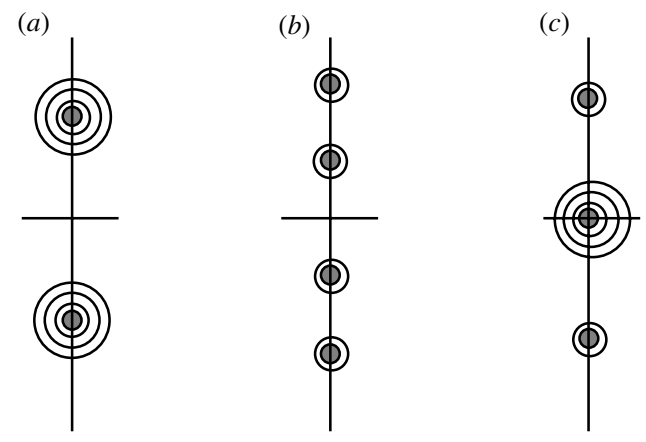

Figure 6. Position of the eigenvalues $\lambda=\mathrm{i} \Omega$ with $\Delta(\Omega, \alpha, \beta)=0$ when the amplitude $|A|$ is zero: (a) the case $\beta=0$; (b) generic case; (c) case where $D_{k} \alpha= \pm D_{\ell} \beta$ when two pairs meet at the origin.

roots of (7.11) coalesce into a degenerate quartic resonance, as shown in figure 6(a). When $D_{k} \alpha= \pm D_{\ell} \beta$, one of the pairs in (7.11) vanishes, and so two pairs coalesce at the origin, as shown in figure $6(c)$. For other values of $k$ and $\ell$, the roots are in the qualitative form shown in figure $6(b)$.

For the case $|A|>0$, the general stability conditions for the quartic (6.4) can be applied to (7.10), but it is easier and more instructive to note that for $|A|$ small the polynomial again factorizes leading to the four expressions for the stability exponents given in (1.10) for transverse instabilities and (1.12) for longitudinal instabilities.

The results of this section are general and apply to any Hamiltonian PDE (which can be cast into multi-symplectic form) with SCWs. The coefficients in the stability quartic require only the dispersion relation, and the parameters $a$ and $b$ associated with the nonlinear correction to the frequency.

\subsection{Derivatives of the frequency and the dispersion relation}

Consider a dispersion relation $D(\omega, k, \ell)=0$ with the hypotheses $D_{\omega} \neq 0, D_{k} \neq 0$ and $D_{\ell} \neq 0$, and treat $\omega$ as a function of $k$ and $\ell$. Then, differentiating $D(\omega(k, \ell), k, \ell)=0$ leads to

$$
\omega_{k}=-\frac{D_{k}}{D_{\omega}}, \quad \omega_{\ell}=-\frac{D_{\ell}}{D_{\omega}} .
$$

Differentiating again then leads to

$$
\begin{aligned}
& D_{\omega} \omega_{k k}+D_{\omega \omega} \omega_{k}^{2}+2 D_{\omega k} \omega_{k}+D_{k k}=0, \\
& D_{\omega} \omega_{k \ell}+D_{\omega \omega} \omega_{k} \omega_{\ell}+D_{\omega k} \omega_{\ell}+D_{\omega \ell} \omega_{k}+D_{k \ell}=0, \\
& D_{\omega} \omega_{\ell \ell}+D_{\omega \omega} \omega_{\ell}^{2}+2 D_{\omega \ell} \omega_{\ell}+D_{\ell \ell}=0 .
\end{aligned}
$$

Combining the above expressions then leads to

$$
\omega_{k k}=\frac{\delta_{1}}{D_{\omega}^{3}}, \quad \omega_{k \ell}=\frac{\delta_{3}}{D_{\omega}^{3}}, \quad \omega_{\ell \ell}=\frac{\delta_{2}}{D_{\omega}^{3}},
$$

where

$$
\delta_{1}=\operatorname{det}\left[\begin{array}{ccc}
D_{\omega \omega} & D_{\omega k} & D_{\omega} \\
D_{k \omega} & D_{k k} & D_{k} \\
D_{\omega} & D_{k} & 0
\end{array}\right], \quad \delta_{2}=\operatorname{det}\left[\begin{array}{ccc}
D_{\omega \omega} & D_{\omega \ell} & D_{\omega} \\
D_{\ell \omega} & D_{\ell \ell} & D_{\ell} \\
D_{\omega} & D_{\ell} & 0
\end{array}\right],
$$


and

$$
\delta_{3}=D_{\omega \ell} D_{k} D_{\omega}+D_{\omega k} D_{\ell} D_{\omega}-D_{\omega \omega} D_{\ell} D_{k}-D_{k \ell} D_{\omega}^{2} .
$$

Combining these expressions leads to the formula

$$
-D_{\omega}^{4} \operatorname{det}\left(\begin{array}{cc}
\omega_{k k} & \omega_{k \ell} \\
\omega_{\ell k} & \omega_{\ell \ell}
\end{array}\right)=\operatorname{det}\left[\begin{array}{cccc}
D_{\omega \omega} & D_{\omega k} & D_{\omega \ell} & D_{\omega} \\
D_{k \omega} & D_{k k} & D_{k \ell} & D_{k} \\
D_{\ell \omega} & D_{\ell k} & D_{\ell \ell} & D_{\ell} \\
D_{\omega} & D_{k} & D_{\ell} & 0
\end{array}\right],
$$

which we have not seen in the literature. The proof of this formula follows from a direct calculation.

The sign of the determinant in (7.13) is independent of the choice of coordinates in the following sense. The dispersion function $D(\omega, k, \ell)$ is not unique. Any other function $E(\omega, k, \ell)$ with the same zeros, say $E(\omega, k, \ell)=d(\omega, k, \ell) D(\omega, k, \ell)$ for some non-vanishing function $d(\omega, k, \ell)$ is also a dispersion function. However, it is easy to show that the determinant (7.13) based on any other dispersion function $E(\omega, k, \ell)$ has the same sign as the determinant based on $D(\omega, k, \ell)$.

\section{Transverse instabilities of weakly nonlinear short-crested water waves}

In this section, expressions for the stability exponents are explicitly computed for weakly nonlinear short-crested gravity water waves on an infinite depth fluid when $\beta$ is small, but outside a neighbourhood of $\beta=0$.

The dispersion relation for water waves is given in (1.17). The coefficient $a$ for water waves is strictly negative, and

$$
\operatorname{det}\left[\begin{array}{cccc}
D_{\omega \omega} & D_{\omega k} & D_{\omega \ell} & D_{\omega} \\
D_{k \omega} & D_{k k} & D_{k \ell} & D_{k} \\
D_{\ell \omega} & D_{\ell k} & D_{\ell \ell} & D_{\ell} \\
D_{\omega} & D_{k} & D_{\ell} & 0
\end{array}\right]=\operatorname{det}\left[\begin{array}{cccc}
2 & 0 & 0 & 2 \omega \\
0 & -\frac{g \ell^{2}}{v^{3}} & \frac{g k \ell}{v^{3}} & -\frac{g k}{v} \\
0 & \frac{g k \ell}{v^{3}} & -\frac{g k^{2}}{v^{3}} & -\frac{g \ell}{v} \\
2 \omega & -\frac{g k}{v} & -\frac{g \ell}{v} & 0
\end{array}\right]=2 \frac{\omega^{6}}{v^{4}}>0 .
$$

The Hessian of $\omega$ with respect to $k$ and $\ell$ has the simple form

$$
\left.\left[\begin{array}{cc}
\omega_{k k} & \omega_{k \ell} \\
\omega_{\ell k} & \omega_{\ell \ell}
\end{array}\right]=\frac{g}{4 \omega v^{3}}\left[\begin{array}{cc}
2 \ell^{2}-k^{2} & -3 k \ell \\
-3 k \ell & 2 k^{2}-\ell^{2}
\end{array}\right]=\frac{\omega}{4 v^{4}}\left[\begin{array}{rr}
\ell & k \\
-k & \ell
\end{array}\right] 2 \begin{array}{rrr}
2 & 0 & -1 \\
0 & -k \\
k & \ell
\end{array}\right]
$$

By applying (1.14) to (8.1), it is immediate that weakly nonlinear SCWs are unstable to long-wave perturbations.

The dependence of the transverse instabilities on the perturbation wavenumbers $(\alpha, \beta)$ is obtained from (1.10). For water waves the stability exponents are $\lambda= \pm \mathrm{i} \Omega$ with

$$
\Omega=\left\{\begin{array}{l}
\frac{g}{2 v \omega}(k \alpha+\ell \beta)-\sigma_{+}|A|+\cdots \\
\frac{g}{2 v \omega}(k \alpha+\ell \beta)+\sigma_{+}|A|+\cdots \\
\frac{g}{2 v \omega}(k \alpha-\ell \beta)-\sigma_{-}|A|+\cdots \\
\frac{g}{2 v \omega}(k \alpha-\ell \beta)+\sigma_{-}|A|+\cdots
\end{array}\right.
$$

with $\sigma_{+}$and $\sigma_{-}$defined in (1.19). 
(a)

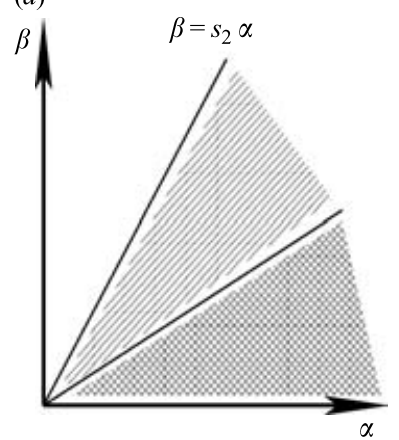

(b)

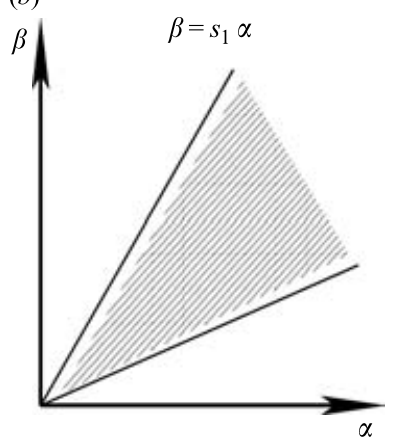

(c)

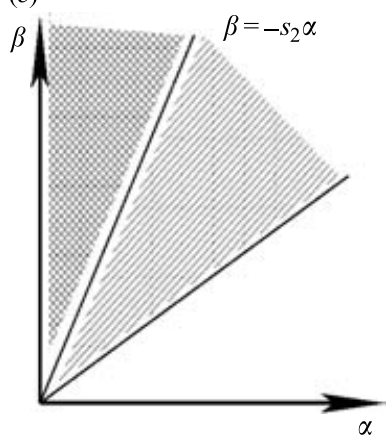

FIGURE 7. Position of unstable modes for each wedge in the $(\alpha, \beta)$-plane when $(a) 0<$ $\ell<1 / \sqrt{2} k$, (b) $1 / \sqrt{2} k<\ell<\sqrt{2} k$, (c) $\ell>\sqrt{2} k$. In terms of the angle of incidence $\theta$ (see (8.3) for a definition) these regions correspond to (a) $54.73^{\circ}<\theta<90^{\circ}$, (b) $35.26^{\circ}<\theta<54.73^{\circ}$, (c) $0<\theta<35.26^{\circ}$.

For all admissible values of $k$ and $\ell$ for SCWs there are unstable wedges in the $(\alpha, \beta)$-plane. However, the properties of these instabilities depend on the values of $k$ and $\ell$. There are three regions in $(k, \ell)$ space and for each of these regions the unstable wedges in the $(\alpha, \beta)$-plane are shown in figure 7 .

For weakly three-dimensional SCWs, that is; when $\ell$ is small (and $\ell^{2}<k^{2} / 2$ ), there are two unstable wedges, a as shown in figure $7(a)$. In the lower unstable wedge, there are two unstable modes and in the middle wedge, there is one unstable mode (as shown in figure 3 ).

In the intermediate region, when $1 / k^{2} 2,<\ell^{2}<2 k^{2}$, there is only one unstable wedge, as shown in figure $7(b)$, and it has only one unstable mode. In the large $\ell$ region, where $\ell^{2}>2 k^{2}$, there are again two unstable wedges with the higher wedge (darker shading) now having two unstable modes, as shown in figure $7(c)$. The location of the unstable wedges in figure $7(c)$ is the reverse of that in figure $7(a)$.

The results in figure 7 can also be interpreted in terms of a wave reflection off a wall. Consider the case of an incident wave of wavelength $2 \pi / v$, where $v=\sqrt{k^{2}+\ell^{2}}$, being fully reflected off a vertical wall. Let $k=v \sin \theta$ and $\ell=v \cos \theta$. Then, $\theta$ is the angle between the direction of propagation of the incident wave and the normal to the wall (cf. Roberts 1983). In terms of the angle $\theta$, the critical values in figure 7 are

$$
\left.\begin{array}{rll}
\ell / k=\sqrt{2} & \Rightarrow & \theta=\tan ^{-1}(1 / \sqrt{2}) \approx 35.26^{\circ}, \\
\ell / k=\frac{1}{\sqrt{2}} & \Rightarrow & \theta=\tan ^{-1}(\sqrt{2}) \approx 54.73^{\circ} .
\end{array}\right\}
$$

The limit $\theta \rightarrow 90^{\circ}$ corresponds to the limit where the two waves are collinear Stokes travelling waves. Hence, figure 7(a) corresponds the the region closest to the travelling, wave limit. Note that this limit is singular so the results are not valid in the limit $\theta \rightarrow 90$. Figure $7(a)$ is consistent with the fact that the Stokes travelling-wave is modulation unstable, but it also shows a difference from the travelling-wave case: the darker shaded region has two modes of instability, whereas a Stokes travelling wave would have only one unstable mode. The limit $\theta \rightarrow 0^{\circ}$ corresponds to the standingwave limit. Near $\theta=0$, the instability regions in figure 7(c) are just the opposite of figure $7(a)$, with the strongest region of instability predominantly in the $y$-direction. This limit is consistent with the modulation instability of pure standing waves; further 
details of the instability of pure standing waves using the multi-symplectic framework can be found in Bridges \& Laine-Pearson (2004).

\section{Longitudinal instabilities of weakly nonlinear short-crested water waves}

When $\beta=0$, the leading-order term for the roots of the stability quartic (7.10) depends also on the terms of order $|A|^{4}$ in the coefficients. When $\beta=0$ and $|A|=0$, the stability quartic (7.10) has a quartic root

$$
\Delta(\Omega, \alpha, \beta)=\frac{1}{|\boldsymbol{\Lambda}|}\left(D_{\omega} \Omega+D_{k} \alpha\right)^{4} .
$$

Hence, the perturbation of this root for $|A| \neq 0$ has a leading-order term of the order of the fourth root of the perturbation. Since the perturbation is of order $|A|^{2}$, the leading-order expansion for the perturbed quartic root is of the form

$$
\Omega=-\frac{D_{k}}{D_{\omega}} \alpha+\Omega_{1}|A|^{1 / 2}+\Omega_{2}|A|+\cdots
$$

However, owing to symmetry, the term $\Omega_{1}=0$ and the leading-order term is of order $|A|$, but the coefficient $\Omega_{2}$ then depends on the terms of order $|A|^{4}$ in the coefficients of (7.10). Let $\delta_{1}$ be as defined in $\S 7.1$, then a calculation shows - to leading order in $|A|-$ that the quartic root associated with $\beta=0$ perturbs into the four roots (expressing the four values of $\Omega_{2}$ as $\pm \mu_{ \pm}$)

$$
\Omega=\left\{\begin{array}{l}
-\frac{D_{k}}{D_{\omega}} \alpha+\mu_{+}|A|+\cdots \\
-\frac{D_{k}}{D_{\omega}} \alpha+\mu_{+}|A|+\cdots \\
-\frac{D_{k}}{D_{\omega}} \alpha-\mu_{-}|A|+\cdots \\
-\frac{D_{k}}{D_{\omega}} \alpha+\mu_{-}|A|+\cdots
\end{array} \quad \text { with } \quad \mu_{ \pm}^{2}=-\frac{(a \pm b)}{D_{\omega}^{4}} \delta_{1} \alpha^{2}\right.
$$

However, in $\S 7.1$ it was shown that $\delta_{1}=\omega_{k k} D_{\omega}^{3}$. Substituting this expression into $\mu_{ \pm}^{2}$ and using the definitions of $\omega_{2}^{T W}$ and $\omega_{2}^{S C W}$ recovers the expressions in (1.13).

The eight stability exponents are given by $\lambda= \pm \mathrm{i} \Omega$, with $\Omega$ taking the four values above. Clearly, stability is determined by the signs of $\mu_{ \pm}^{2}$, and the signs of $\mu_{ \pm}^{2}$ are determined by the signs of $(a+b),(a-b)$ and $\delta_{1}$. With $\bar{k}=v \sin \theta$ and $\ell=v \cos \theta$, the coefficients $a$ and $b(=\Upsilon$ in (4.3) in the SCW limit) can be expressed in the form

$$
\begin{aligned}
& a=-4 g v^{2}, \\
& b=4 g v^{2}\left(-2+6 \cos ^{2} \theta+\frac{8 \cos ^{4} \theta}{\sin \theta-2}+2 \cos ^{4} \theta\right) .
\end{aligned}
$$

The normalized (divided by $\left.4 g v^{2}\right)$ expressions for $(a+b)$ and $(a-b)$ are shown in figure 8.

However, longitudinal stability is determined by the the product of $(a \pm b)$ with $\delta_{1}\left(\right.$ or $\left.\omega_{k k}\right)$. The sign of $\delta_{1}$ is determined by the sign of $2 \ell^{2}-k^{2}$ and this function is positive for $\theta$ small and changes sign when $\theta \approx 54.74^{\circ}$. Therefore, longitudinal instability for $|A|$ small is determined by the inequalities

$$
(a+b)\left(k^{2}-2 \ell^{2}\right)<0 \text { or }(a-b)\left(k^{2}-2 \ell^{2}\right)<0 .
$$




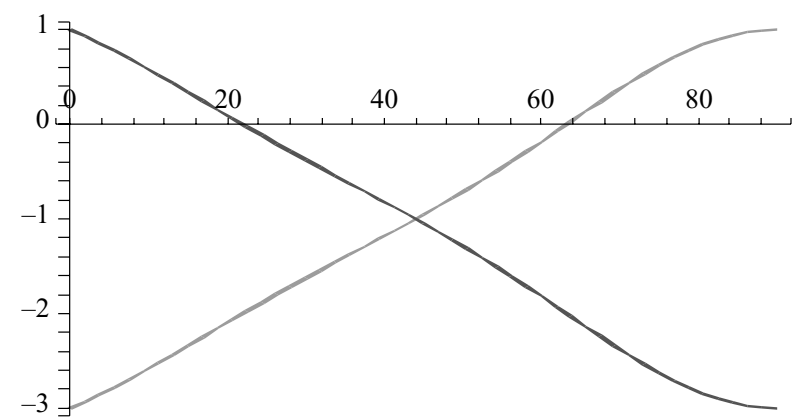

FIgURE 8. Plot of $(a+b)$ and $(a-b)$, normalized by $4 g\left(k^{2}+\ell^{2}\right)$, as functions of $\theta$. The decreasing function is $(a+b)$ and it passes through zero when $\theta \approx 21.96^{\circ}$. The function $(a-b)$ vanishes when $\theta \approx 63.26^{\circ}$.

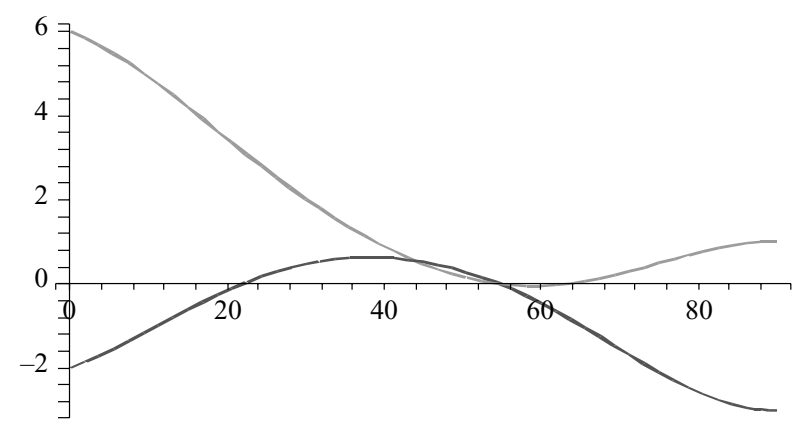

Figure 9. Plot of $(a+b)\left(k^{2}-2 \ell^{2}\right)$ (the predominantly lower curve) and $(a-b)\left(k^{2}-2 \ell^{2}\right)$ as functions of $\theta$.

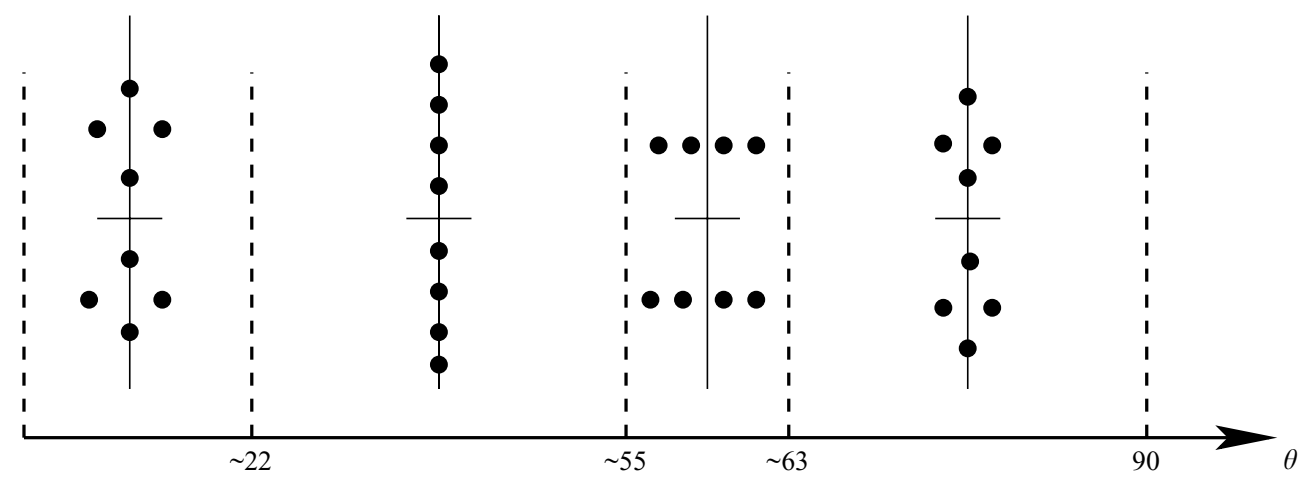

FIGURE 10. Schematic position in the complex $\lambda$-plane of the eigenvalues associated with longitudinal instability for each $\theta$ region.

The two functions in these inequalities are plotted as functions of $\theta$ in figure 9. Instability is signalled when either of the functions in figure 9 is negative.

There are four distinct $\theta$ regions separated by the zeros of $(a+b),(a-b)$ and $\delta_{1}$, as shown in figure 10 . In the region $0<\theta<22^{\circ}$, there is one (complex conjugate) pair of unstable eigenvalues; for $22^{\circ}<\theta<55^{\circ}$, all roots are stable. In the region $55^{\circ}<\theta<63^{\circ}$,

\section{CAMBRIDGE JDURNALS}


there are two pairs of unstable eigenvalues, and then in the region $63^{\circ}<\theta<90^{\circ}$, there is one pair of unstable eigenvalues.

The two unstable regions $0<\theta<22^{\circ}$ and $63^{\circ}<\theta<90^{\circ}$ agree with the predictions of Roskes $(1976 b)$. The angle used by Roskes is equal to $2(90-\theta)$, so $\theta \approx 22^{\circ}$ corresponds to Roskes' $136^{\circ}$ and $\theta \approx 63^{\circ}$ corresponds to Roskes' $55^{\circ}$. However, his analysis misses the unstable region $55^{\circ}<\theta<63^{\circ}$ because both $\mu_{ \pm}^{2}$ are negative in that region.

The qualitative position of the roots for $\beta=0$ will persist for $\beta$ small. Therefore, we expect the position of the roots in figure 10 to persist in a small wedge around the axis $\beta=0$. This small wedge of longitudinal instabilities is consistent with the numerical calculations reported in figures 8 and 9 in Ioualalen \& Kharif (1994).

\subsection{Instability of short-crested capillary-gravity water waves}

The addition of capillarity brings new features that are worthy of mention. The issues are just sketched in this subsection. The first new feature is that a rigorous theory of existence for capillary-gravity SCWs - and their deformation into the non-resonant two-wave interaction - is available (Craig \& Nicholls 2000), suggesting that they are more robust than pure gravity waves. Indeed, it is conceivable that a rigorous theory for instability could also be developed for the case of capillary-gravity SCWs. The second issue is the larger range of possibilities for instability. For example, all the critical coefficients (the determinant (8.1), and the parameters $\sigma_{+}^{2}, \sigma_{-}^{2} \mu_{-}^{2}$ and $\mu_{+}^{2}$ ) have additional sign changes in parameter space. A clue to the range of possibilities for transverse instabilities can be seen from the range of instabilities of oblique capillarygravity travelling waves (cf. $\S 5$ of Bridges 1996). When capillary forces are added, the longitudinal instabilities will also change. For example, it is shown inBridges, Dias \& Menasce (2001) that the coefficients $a$ and $b$ have sign changes along lines in the $(\theta, \tau)$ plane where $\tau$ is the Bond number.

\section{Concluding remarks}

Two directions in which the theory here can be extended are the stability of SCWs in shallow water, and the stability of the resonant and non-resonant three-wave interaction.

The issue with shallow-water and finite-depth SCWs is the mean flow which can influence stability. However, the theory presented here can be combined with the theory in Bridges et al. (2001). The idea would be to couple the six-parameter two-wave interaction with the three-parameter mean flow in Bridges et al. (2001), leading to a nine-parameter problem. The stability polynomial will be a sixth-order polynomial in this case. Theoretically the strategy is clear, but the details will be lengthy for this case. There are questions to be tackled, however. It would be of great interest to have analytical results for weakly nonlinear SCWs in shallow water, and also there are open questions about the stability of two-wave interactions in shallow water (cf. Onorato et al. (2002)).

The resonant and non-resonant three-wave interaction is the next logical step. There is enough symmetry (when the three waves are oblique), so the idea of embedding the resonant interaction in a three-phase non-resonant interaction still makes theoretical sense. In infinite depth this will be a nine-parameter problem, and the stability polynomial will be sixth order. In finite depth, when mean flow effects are accounted for, there will be twelve parameters, and the stability polynomial will be eighth order. Some results on the weakly nonlinear three-wave interaction of water waves in infinite depth have been obtained by Laine-Pearson (2002). 
The authors are grateful to all three referees for their constructive and useful suggestions, and particularly grateful to the referee who encouraged the authors to examine more closely the nature of the limit $\beta=0$ in the linear stability problem.

\section{Appendix. Some properties of short-crested wave solutions of multi-symplectic PDEs}

What precisely is a short-crested Stokes wave? Key features are $(a)$ periodicity in all space directions and time; $(b)$ uniformly travelling; $(c)$ reflection symmetric in the direction orthogonal to the direction of propagation. In the multi-symplectic setting, the concept of a reversor is used to give a precise definition.

A solution, $\widehat{Z}(x, y, t)$ of $(3.1)$, is called a short-crested wave if it is periodic in $x, y$ and $t$, is travelling in the $x$-direction (depends on $x$ and $t$ in linear combination only) and satisfies $\mathscr{R} \widehat{Z}(x, y, t)=\widehat{Z}(x, y, t)$.

An immediate consequence of this definition is that the transverse momentum of a short-crested wave is identically zero. An application of Noether's theorem shows that the appropriate form for the density of the momentum vector is

$$
\mathscr{M}=\left(\frac{1}{2}\left\langle\mathbf{M} Z_{x}, Z\right\rangle, \frac{1}{2}\left\langle\mathbf{M} Z_{y}, Z\right\rangle\right) .
$$

This form can be verified by using Noether's theorem, as in the Appendix of Bridges \& Laine-Pearson (2004), or by direct calculation as below.

For functions satisfying (3.1), we have the following conservation law for the transverse momentum

$$
\frac{\partial}{\partial t}\left(\frac{1}{2}\left\langle\mathbf{M} Z_{y}, Z\right\rangle\right)+\frac{\partial}{\partial x}\left(\frac{1}{2}\left\langle\mathbf{K} Z_{y}, Z\right\rangle\right)+\frac{\partial}{\partial y}\left(S(Z)-\frac{1}{2}\left\langle\mathbf{M} Z_{t}, Z\right\rangle-\frac{1}{2}\left\langle\mathbf{K} Z_{x}, Z\right\rangle\right)=0,
$$

and soon a space of functions that are periodic in $x$ and $y$ (and normalizing the period to $2 \pi$ in each direction)

$$
\mathscr{M}_{y}=\int_{\mathbb{T}^{2}} \frac{1}{2}\left\langle\mathbf{M} Z_{y}, Z\right\rangle \mathrm{d} x \mathrm{~d} y=\frac{1}{(2 \pi)^{2}} \int_{0}^{2 \pi} \int_{0}^{2 \pi} \frac{1}{2}\left\langle\mathbf{M} Z_{y}, Z\right\rangle \mathrm{d} x \mathrm{~d} y
$$

is conserved in time. For water waves, this functional can be expressed in the form

$$
\mathscr{M}_{y}=\int_{\mathbb{T}^{2}}-\Phi \eta_{y} \mathrm{~d} x \mathrm{~d} y \text {. }
$$

One implication of the above definition of SCWs is that it implies immediately that $\mathscr{M}_{y}$ - when evaluated on an SCW - is identically zero for all time. This follows since

$$
\begin{aligned}
\mathscr{M}_{y}(\mathscr{R} Z)= & \int_{\mathbb{T}^{2}} \frac{1}{2}\left\langle\mathbf{M R}(Z(x,-y, t))_{y}, \mathbf{R} Z(x,-y, t)\right\rangle \mathrm{d} x \mathrm{~d} y \quad \text { (by definition), } \\
= & -\int_{\mathbb{T}^{2}} \frac{1}{2}\left\langle\mathbf{M R} Z_{y}(x,-y, t), \mathbf{R} Z(x,-y, t)\right\rangle \mathrm{d} x \mathrm{~d} y \\
= & \left.-\int_{\mathbb{T}^{2}} \frac{1}{2}\left\langle\mathbf{R} \mathbf{M} Z_{y}(x,-y, t), \mathbf{R} Z(x,-y, t)\right\rangle \mathrm{d} x \mathrm{~d} y \quad \text { (using } \mathbf{R M}=\mathbf{M R}\right), \\
= & -\int_{\mathbb{T}^{2}} \frac{1}{2}\left\langle\mathbf{M} Z_{y}(x,-y, t), Z(x,-y, t)\right\rangle \mathrm{d} x \mathrm{~d} y \\
& \left(\operatorname{since}_{\mathbf{n}} \mathbf{R} \operatorname{preserves} \text { the inner product)},\right. \\
= & -\int_{\mathbb{T}^{2}} \frac{1}{2}\left\langle\mathbf{M} Z_{y}(x, y, t), Z(x, y, t)\right\rangle \mathrm{d} x \mathrm{~d} y \\
& (\text { transforming } y \mapsto-y \text { and using periodicity), } \\
= & -\mathscr{M}_{y}(Z) .
\end{aligned}
$$

Therefore, if $\widehat{Z}(x, y \in t)$ is ans $S C W$ and so $\mathscr{R} \widehat{Z}=\widehat{Z}$, it is immediate that $\mathscr{M}_{y}(\widehat{Z})=0$. 


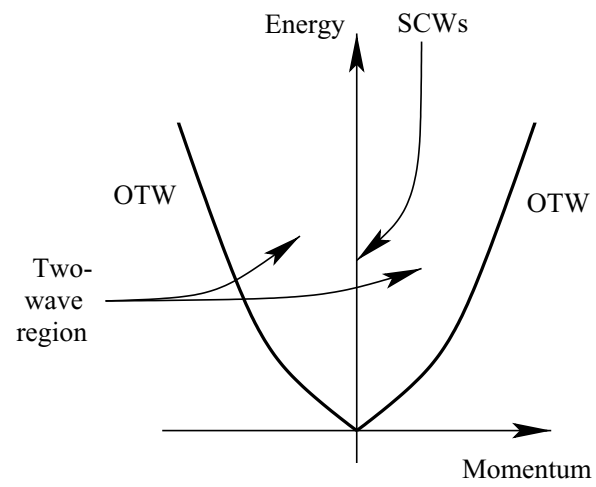

FIGURE 11. Location of SCWs and the two-wave interaction in the energy-(transverse)momentum diagram.

The energy and transverse momentum are useful for distinguishing SCWs from oblique travelling waves (OTW). OTWs have non-zero transverse momentum. A schematic of the energy-momentum (transverse) space is shown in figure 11. The two OTWs correspond to the arms shown, with SCWs along the vertical (zero-momentum) axis. The oblique two-wave interaction then fills out the space between the OTWs and SCWs. The embedding of SCWs in the two-phase wavetrain extends the SCWs into this two-wave region.

This energy momentum diagram is very similar to the energy-momentum diagram for the spherical pendulum and for standing waves (cf. Bridges \& Laine-Pearson 2004).

\section{REFERENCES}

Ablowitz, M. J. \& Benney, D. J. 1970 The evolution of multi-phase modes for nonlinear dispersive waves. Stud. Appl. Maths 49, 225-238.

Badulin, S. I., Shira, V. I., Kharif, C. \& Ioualalen, M. 1995 On two approaches to the problem of instability of short-crested water waves. J. Fluid Mech. 303, 297-326.

BRIDGES, T. J. 1996 Periodic patterns, linear instability, symplectic structure and mean-flow dynamics for three-dimensional surface waves. Phil. Trans. R. Soc. Lond. A 354, 533-74.

Bridges, T. J. 1997a Multi-symplectic structures and wave propagation. Math. Proc. Camb. Phil. Soc. 121, 147-190.

BRIDGES, T. J. 1997b A geometric formulation of the conservation of wave action and its implications for signature and the classification of instabilities. Proc. R. Soc. Lond. A 453, 1365-1395.

BRIDGES, T. J. 1998 Toral-equivariant partial differential equations and quasiperiodic patterns. Nonlinearity 11, 467-500.

BRIDGES, T. J. 2001 Transverse instability of solitary-wave states of the water-wave problem. J. Fluid Mech. 439, 255-278.

Bridges, T. J., Dias, F. \& Menasce, D. 2001 Steady three-dimensional water-wave patterns on a finite-depth fluid. J. Fluid Mech. 436, 145-175.

Bridges, T. J. \& Laine-Pearson, F. E. 2001 Multisymplectic relative equilibria, multiphase wavetrains and coupled NLS equations. Stud. Appl. Maths 107, 137-155.

Bridges, T. J. \& LAINE-PeARson, F. E. 2004 Nonlinear counterpropagating waves, multi-symplectic geometry, and the instability of standing waves. SIAM J. Appl. Maths 64, 2096-2120.

Craig, W. \& Nicholls, D. P. 2000 Traveling two and three dimensional capillary-gravity water waves. SIAM J. Math. Anal. 32, 323-359.

Craig, W. \& Nicholls, D. P. 2002 Traveling gravity water waves in two and three dimensions. Eur. J. Mech. B/Fluids 21, 615-641.

Elfouhaily, T., Thompson, D. R., Vandemark, D. \& Chapron, B. 2000 Truncated Hamiltonian Cambrsus surface perturbation in nonlinear wave theories. Waves Random Media 10, 103-116. 
Hsu, J. R. C., Silvester, R. \& Tsuchiya, Y. 1980 Boundary-layer velocities and mass transport in short-crested waves. J. Fluid Mech. 99, 321-342.

IouAlalen, M. 1993 Fourth-order approximation of short-crested waves. C. R. Acad Sci. Paris 316, $1193-1200$.

IOUALALEN, M. \& Kharif, C. 1994 On the subharmonic instabilities of steady three-dimensional deep water waves. J. Fluid Mech. 262, 265-291.

Kimmoun, O., Branger, H. \& Kharif, C. 1999 On short-crested waves: experimental and analytical investigations. Eur. J. Mech. B/Fluids 18, 889-930.

Knobloch, E. \& GibBon, J. D. 1991 Coupled NLS equations for counter propagating waves in systems with reflection symmetry. Phys. Lett. A 154, 353-356.

KuKsin, S. 2000 Analysis of Hamiltonian PDEs. Oxford University Press.

Laine-Pearson, F. E. 2002 Geometry and Stability of Multi-periodic Surface Wave Patterns. PhD thesis, University of Surrey.

LanCaster, P. 1966 Lambda Matrices and Vibrating Systems. Pergamon.

Longuet-Higgins, M. S. 1962 Resonant interactions between two trains of gravity waves. J. Fluid Mech. 12, 321-32.

Milewski, P. A. \& Keller, J. B. 1996 Three-dimensional water waves. Stud. Appl. Maths 100, 95-106.

Mollo-Christensen, E. 1981 Modulational instability of short-crested Stokes waves. Phys. Fluids 24, 775-776.

Onorato, M., Ambrosi, D., Osborne, A. R. \& Serio, M. 2003 Interaction of two quasimonochromatic waves in shallow water. Phys. Fluids 15, 3871-3874.

Pierce, R. D. \& Knobloch, E. 1994 On the modulational instability of travelling and standing water waves. Phys. Fluids 6, 1177-1190.

PIERSON JR, W. J. 1993 Oscillatory third-order perturbation solutions for sums of interacting long-crested Stokes waves on deep water. J. Ship Res. 37, 354-383.

RoberTs, A. J. 1983 Highly nonlinear short-crested water waves. J. Fluid Mech. 135, 301-321.

Roberts, A. J. \& Peregrine, D. H. 1983 Notes on long-crested water waves. J. Fluid Mech. 135, $325-335$.

Roskes, G. J. 1976 Some nonlinear multiphase wavetrains. Stud. Appl. Maths 55, 231-238.

Roskes, G. J. 1976 Nonlinear multiphase deep-water wavetrains. Phys. Fluids 19, 1253-1254.

Smith, J. M. \& Vincent, C. L. 1992 Shoaling and decay of two wave trains on a beach. J. Waterways Port Coast \& Ocean Engng 118, 517-533.

Weber, B. L. \& BARrick, D. E. 1977 On the nonlinear theory for gravity waves on the ocean's surface. I. Derivations, II. Interpretations and applications. J. Phys. Ocean. 7, 3-10, 11-21.

Whitham, G. B. 1974 Linear and Nonlinear Waves. Wiley-Interscience.

Willebrand, J. 1975 Energy transport in a nonlinear and inhomogeneous random gravity wave field. J. Fluid Mech. 70, 113-26.

ZakHaRov, V. E. 1968 Stability of periodic waves of finite amplitude on the surface of a deep fluid. Zh. Prikl. Mekh. Fiz. 9, 86-94 (English trans: J. Appl. Mech. Tech. Phys. 2, 190). 